Research Article

\title{
Identification and Analysis of Components in Yizhi Granule and Cynomolgus Monkey Plasma after Oral Administration by UPLC/ ESI-Q-TOF MS and Their Protective Effects on PC12 Cells
}

\author{
Erwei Hao, ${ }^{1,2,3}$ Jianfeng Qin $\mathbb{D}^{1,2}$ Wei Wei, ${ }^{1,2}$ Jianhua Miao $\mathbb{D}^{3}{ }^{3}$ Yan Xie, ${ }^{1,2}$ \\ Xianglong Pan, ${ }^{1,2}$ Hangxuan Wu, ${ }^{1,2}$ Jinling Xie, ${ }^{1,2}$ Xiaosu Fan, ${ }^{4}$ Zhengcai Du $\mathbb{D},{ }^{1,2,3}$ \\ Xiaotao Hou $\mathbb{D}^{1,2,3}$ and Jiagang Deng $\mathbb{D i D}^{1,2,3}$ \\ ${ }^{1}$ Guangxi Key Laboratory of Efficacy Study on Chinese Materia Medica, Guangxi University of Chinese Medicine, Nanning, \\ Guangxi 530200, China \\ ${ }^{2}$ Collaborative Innovation Center for Research on Functional Ingredients of Agricultural Residues, \\ Guangxi University of Chinese Medicine, Nanning, Guangxi 530200, China \\ ${ }^{3}$ Postdoctoral Workstation, Guangxi Institue of Medicinal Plants, Nanning 530023, China \\ ${ }^{4}$ Experimental Center of College of Agriculture, Guangxi University, Nanning 530005, China
}

Correspondence should be addressed to Zhengcai Du; duzhengcai8@163.com, Xiaotao Hou; xthou@126.com, and Jiagang Deng; dengjg53@126.com

Received 17 December 2019; Accepted 28 January 2020; Published 8 April 2020

Academic Editor: Luca Tortora

Copyright (c) 2020 Erwei Hao et al. This is an open access article distributed under the Creative Commons Attribution License, which permits unrestricted use, distribution, and reproduction in any medium, provided the original work is properly cited.

\begin{abstract}
Yizhi Granule (YZG) is a health food containing six traditional Chinese medicines (TCMs). It improves memory barriers in rat experiments. Here, we describe the first fast and sensitive ultraperformance liquid chromatography/electrospray ionization quadrupole time-of-flight mass spectrometry (UPLC/ESI-Q-TOF MS) method for analyzing YZG in plasma. We used this technique for studies in cynomolgus monkey plasma. By comparing retention time, MS, and MS/MS data of reference compounds, 70 compounds were detected in YZG. Of these, 63 were identified including 60 saponins, 2 flavones, and 1 methyl ester. There were 33 saponins, 1 flavone, and 1 methyl ester in the plasma. Next, to study the therapeutic properties of YZG, the neuroprotective effect of some of the absorbed components was evaluated using PC12 cell damage caused by the A $\beta_{25-35}$ model. The results showed that 9 compounds protect PC12 cells from $\mathrm{A} \beta_{25-35}$ with cell viability (\%) of 111.00 \pm 8.12 (G-Rb1), $102.20 \pm 4.22$ (G-Rb2), $100.34 \pm 6.47$ (G-Rd), $102.83 \pm 2.10$ (G-Re), 101.68 \pm 7.64 (NG-Fa), $101.19 \pm 7.83$ (NG-R1), $102.53 \pm 0.55$ (NG-R2), $106.88 \pm 4.95$ (gypenoside A), and 103.95 \pm 4.11 (gypenoside XLIX), respectively, versus the control group (87.51 \pm 6.59 ). These results can reveal the real pharmacodynamic basis of YZG and provide a theoretical basis for subsequent studies. It can also provide some references for the research of Alzheimer's disease.
\end{abstract}

\section{Introduction}

Alzheimer's disease $(\mathrm{AD})$ is a common chronic progressive neurodegenerative disease leading to memory impairment, hypophrenia, behavioral personality degeneration, disability, and premature death [1-3]. The prevalence of $\mathrm{AD}$ in China was $3.21 \%$ among people aged 65 years and older [4], and more than 7 million Chinese people live with AD today [5]. $\mathrm{AD}$ is not only a serious health problem for the elderly but also a severe social problem. It is of global concern. Therefore, developing new drugs to prevent and treat $\mathrm{AD}$ is critical. The amyloid hypothesis is the dominant model of $\mathrm{AD}$ pathogenesis and guides the development of potential treatments. All $\mathrm{AD}$ patients undergo progressive $\beta$-amyloid deposition followed by surrounding neuritic and glial cytopathology in brain regions serving memory and cognition [6].

Traditional Chinese medicine (TCM) plays a vital role in treating Alzheimer's disease in China. It is an essential 
source for new drug development. Many components of Chinese herbs such as ginsenoside $\operatorname{Re}(\mathrm{G}-\mathrm{Re})$ [7], G-Rg3 [8], Baicalin, G-Rb1 [9], G-Rg1 [10], and G-Rf [11] were studied and shown to have an active function on treating AD. YZG is a health food composing 6 TCMs, including Panax ginseng C.A. Mey, Panax notoginseng (Burk) F. H. Chen, Gynostemma pentaphyllum (Thunb.) Makino, Epimedium brevicornu Maxim., Alpinia oxyphylla Miq., and Morus alba L. It was approved as a health food by the CFDA with 2 Chinese patents issued in December 2012.

We recently showed that YZG improved memory barriers in animal experiments, and YZG could protect the PC12 from the damage induced by protein $\mathrm{A} \beta_{25-35}$ [12-15]; however, the active ingredients remain unclear [16]. Although TCMs contain many components, only some are absorbed into the blood for biological activity. Therefore, components found in the plasma are likely to be the most active.

Cynomolgus monkeys are nonhuman primates and are similar to humans in genetics and pathophysiology; thus, they are a useful preclinical model $[17,18]$. Therefore, we used this model to evaluate the TCMs.

TCMs exert their effects through multiple components. Analysis of the chemical composition is a crucial step to understand the therapeutic properties of TCMs. Ultraperformance liquid chromatography-mass spectrometry (UPLC-MS) method has become a dominant tool to analyze the chemical components of TCMs because it offers high speed, wide measurable mass range, high ratio of the resolution, and the capacity for simultaneous qualitative analysis, which is also widely applied in the analysis of in vivo metabolites. For instance, UPLC-QTOF-MS technique was used to identify the absorbed constituents and their metabolic products in rat biosamples [19], and in their following study, an ESI/APCI multimode ionization source was used for LC-MS analysis in order to identify saponin glycosides and saponin aglycones in a single run [20]. In other studies, based on the LC-DAD-MS and UPLC-DAD-QTOF-MS methods, chemical composition, metabolism, and pharmacokinetic studies of herbal medicines were also conducted $[21,22]$. Hence, we established a UPLC/ESI-Q-TOF MS method for the analysis of the chemical composition of YZG and the absorbed ingredients in the plasma of cynomolgus monkeys. Furthermore, the AD cell model induced by $\beta$-amyloid was used to test the protective effects of the absorbed components on nerve cells. The experimental design and workflow of this study were summarized in Figure 1.

\section{Materials and Methods}

2.1. Chemicals, Reagents, and Samples. UPLC-grade acetonitrile was purchased from Merck (Darmstadt, Germany). Formic acid was purchased from Sigma-Aldrich (Mo, USA). Leucine enkephalin was obtained from Waters Corporation (Milford, MA, USA). Distilled water was obtained from Watson's Food \& Beverage (Guangzhou, China).

The PC12 cells were purchased from Jiangsu KeyGEN BioTECH Corp., Ltd (Nanjing, China). Ginsenoside Rb1, ginsenoside $\mathrm{Rb} 2$, ginsenoside $\mathrm{Rb} 3$, ginsenoside $\mathrm{Rc}$, ginsenoside Rd, ginsenoside Re, ginsenoside Rf, ginsenoside Rg1, notoginsenoside R1, 20(S)-notoginsenoside R2, notoginsenoside $\mathrm{Fa}$, gypenosides $\mathrm{A}$, and gypenosides XLIX were purchased from Chengdu Must Bio-Technology Co., Ltd. (Chengdu, China). The 20(S)-ginsenoside Rb2, galantamine $\mathrm{HBr}$, and berberine were purchased from the National Institutes for Food and Drug Control (Beijing, China). The structure of these compounds is shown in Figure 2. Dulbecco's modified Eagle medium (DMEM) and phosphate buffer saline (PBS) were purchased from Jiangsu KeyGEN BioTECH Corp., Ltd. (Nanjing, China). Fetal bovine serum (FBS), trypsin, and dimethyl sulfoxide (DMSO) were purchased from Gibco (Thermo Fisher Scientific, Inc., Waltham, MA, USA). Amyloid $\beta$-protein fragment 25-35 was purchased from Sigma-Aldrich (St. Louis, MO, USA). YZG was produced by Guangxi Wanshoutang Pharmaceutical Co., Ltd., and formulated by water into suspension.

2.2. Animals and Treatments. Six healthy male cynomolgus monkeys (Macaca fascicularis; 7 years old, $7.0 \pm 1.0 \mathrm{~kg}$ ) were obtained from Guangxi cynomolgus medicine applied engineering technology research center (Guangxi province). All experiments were conducted in accordance with the Regulations of Experimental Animal Administration issued by the State Commission of Science and Technology of the People's Republic of China. Experimental animal protocols were approved by the Animal Ethics Committee of the Guangxi University of Chinese Medicine, and all procedures were following the relevant regulations and guidelines.

Each monkey was housed in a suspended stainless steel cage and was maintained under a standard $12 \mathrm{~h}$ light $/ 12 \mathrm{~h}$ dark cycle with free access to water. Animal rooms were kept at $24-26^{\circ} \mathrm{C}$ and relative humidity of $50 \%-70 \%$. A certified primate pellet diet was provided to the monkeys three times each day before the experiments. Fruits were supplemented regularly for nutrition as is standard practice.

2.3. Plasma Sample Preparation. Six cynomolgus monkeys were fasted, except water, for 12 hours. Each monkey was orally administered YZG at a dose of $79.6 \mathrm{~g} / \mathrm{kg}$ body weight. $2 \mathrm{~mL}$ of blood was collected by venipuncture $2 \mathrm{~h}$ after dosing via intragastric gavage. The blood was then centrifuged for $10 \mathrm{~min}$ at $3000 \mathrm{rpm} / \mathrm{min}$ at $4^{\circ} \mathrm{C}$. Methanol $(3 \mathrm{~mL})$ was put in the plasma and vortexed for $1 \mathrm{~min}$ and then was centrifuged for $10 \mathrm{~min}$ at $12000 \mathrm{rpm} / \mathrm{min}$ at $4^{\circ} \mathrm{C}$. The supernatant was purified by solid-phase extraction. The purified liquid was dried under nitrogen gas at $45^{\circ} \mathrm{C}$. The residues were dissolved in $100 \mu \mathrm{L}$ of $70 \%$ methanol and then centrifuged at $12000 \mathrm{rpm} / \mathrm{min}$ for $10 \mathrm{~min}$ at $4^{\circ} \mathrm{C}$; the supernatant was used as the plasma sample.

\subsection{Instrument and Conditions}

2.4.1. Chromatographic Analysis. The separation process was performed by the Waters ACQUITY UPLC I-Class system (Waters Corporation, Milford, MA, USA) with the 


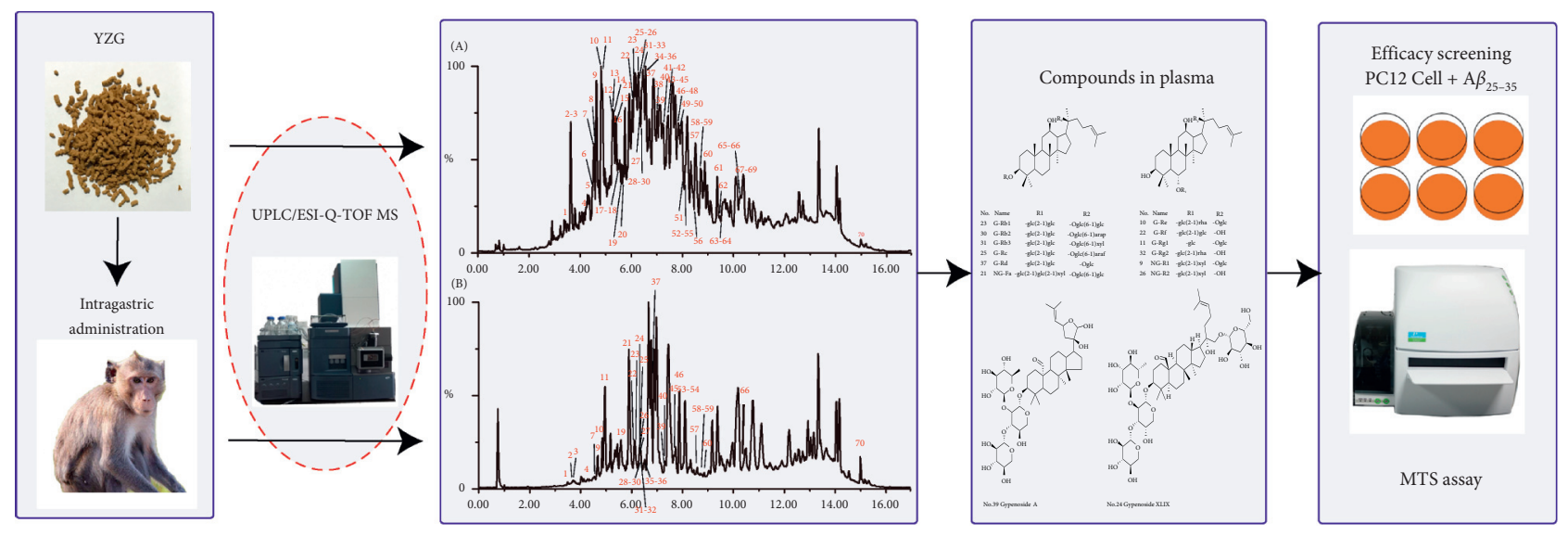

FIgURE 1: Experimental design and workflow in this study.

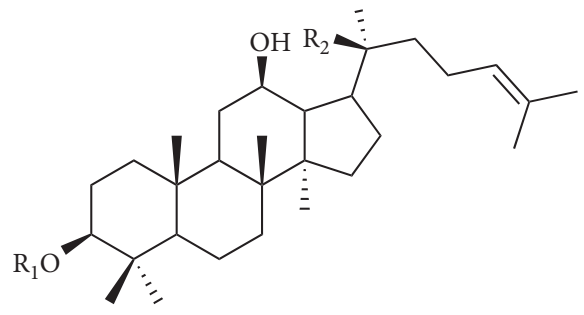

\begin{tabular}{|c|c|c|c|}
\hline No. & Name & R1 & R2 \\
\hline 23 & G-Rb1 & $-\operatorname{glc}(2-1) \operatorname{glc}$ & $-\operatorname{Oglc}(6-1) \operatorname{glc}$ \\
\hline 30 & $\mathrm{G}-\mathrm{Rb} 2$ & $-\operatorname{glc}(2-1) \operatorname{glc}$ & -Oglc(6-1)arap \\
\hline 31 & G-Rb3 & $-\operatorname{glc}(2-1) \operatorname{glc}$ & $-\operatorname{Oglc}(6-1) x y l$ \\
\hline 25 & G-Rc & $-\operatorname{glc}(2-1) \operatorname{glc}$ & $-\operatorname{Oglc}(6-1) \operatorname{araf}$ \\
\hline 37 & G-Rd & $-\operatorname{glc}(2-1) \operatorname{glc}$ & - Oglc \\
\hline & $\mathrm{NG}-\mathrm{Fa}$ & $-\operatorname{glc}(2-1) \operatorname{glc}(2-1) x y l$ & $-\mathrm{Oglc}(6-1) \mathrm{glc}$ \\
\hline
\end{tabular}

(a)

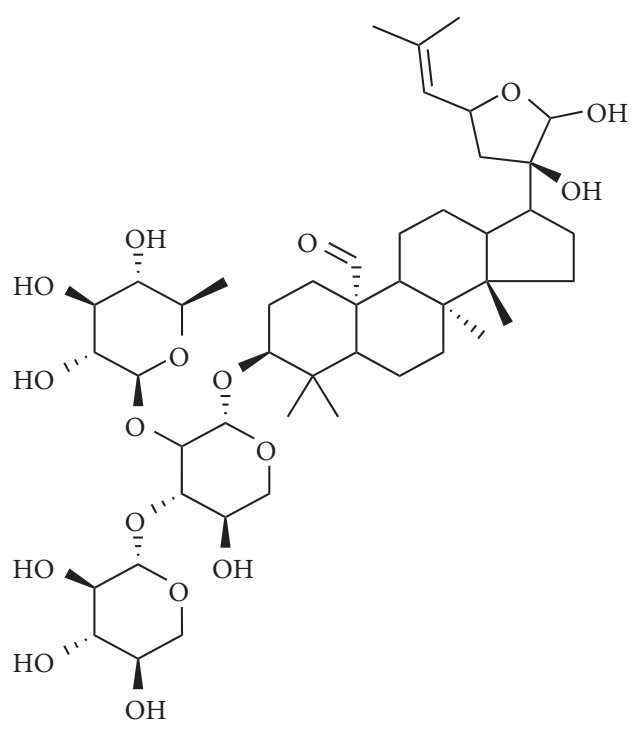

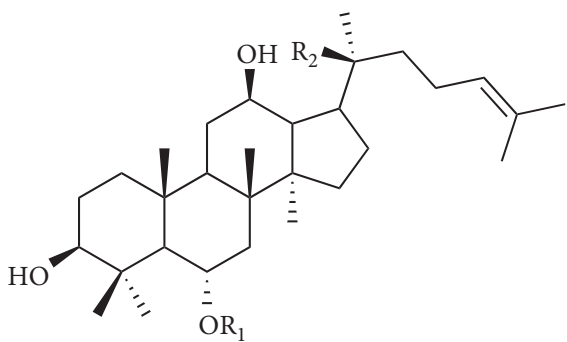

$\begin{array}{lccc}\text { No. } & \text { Name } & \text { R1 } & \text { R2 } \\ 10 & \text { G-Re } & -\operatorname{glc}(2-1) r h a & -\mathrm{Oglc} \\ 22 & \text { G-Rf } & -\operatorname{glc}(2-1) \operatorname{glc} & -\mathrm{OH} \\ 11 & \text { G-Rg1 } & - \text { glc } & -\mathrm{Oglc} \\ 32 & \text { G-Rg2 } & -\operatorname{glc}(2-1) \mathrm{rha} & -\mathrm{OH} \\ 9 & \text { NG-R1 } & -\operatorname{glc}(2-1) \times y l & -\mathrm{Oglc} \\ 26 & \text { NG-R2 } & -\operatorname{glc}(2-1) \times y l & -\mathrm{OH}\end{array}$

(b)

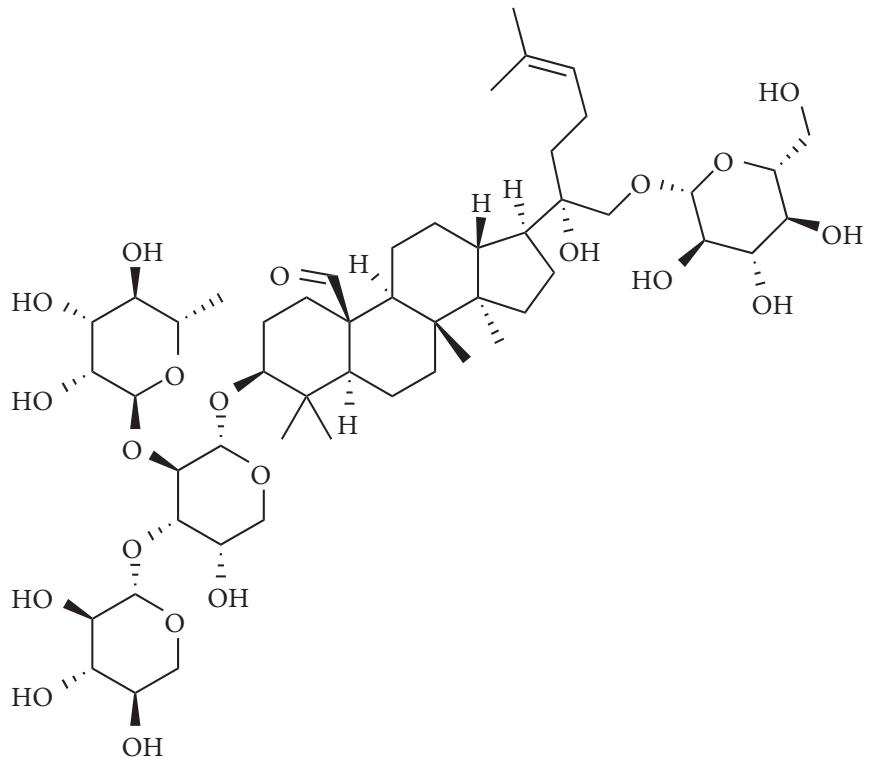

No.39 Gypenoside A

No.24 Gypenoside XLIX

FIgURE 2: Chemical structures of the possible effective compounds of YZG. 
controlled software of Masslynx V4.1. The mobile phase consisted of solvent $\mathrm{A}\left(\mathrm{HCOOH}: \mathrm{H}_{2} \mathrm{O}=0.1: 100, v / v\right)$ and solvent $\mathrm{B}\left(\mathrm{CH}_{3} \mathrm{CN}\right)$; the gradient eluting procedure was as follows: $0-1 \mathrm{~min}, 10 \% \mathrm{~B} ; 1-14 \mathrm{~min}, 10 \%-100 \% \mathrm{~B}$; and $14-17 \mathrm{~min}, 100 \% \mathrm{~B}$ at a flow rate of $0.3 \mathrm{~mL} / \mathrm{min}$. The volume of the sample solution injected into the chromatographic system was $1 \mu \mathrm{L}$, and all the separations were performed at $40^{\circ} \mathrm{C}$.

2.4.2. MS Conditions. The MS analysis was performed by the Xevo G2-XS time-of-flight mass spectrometer coupled with an ACQUITY UPLC I-Class system (Waters Corporation, Milford, MA, USA). The ESI-MS/MS experiment was operated in $\mathrm{MS}^{\mathrm{E}}$ mode to obtain fragmentation in the negative mode. MS conditions were optimized as follows: the acquisition mass range was from 100 to $1500 \mathrm{Da}$ with a $0.5 \mathrm{~s}$ scan time; capillary voltage was $2.0 \mathrm{kV}$; sampling cone voltage was $50.0 \mathrm{~V}$; source temperature was $100^{\circ} \mathrm{C}$; desolvation temperature was $350^{\circ} \mathrm{C}$; cone gas flow was $50.0 \mathrm{~L} /$ $\mathrm{Hr}$; desolvation gas flow was $700.01 / \mathrm{Hr}$. The data were collected on a continuum, and the mass was corrected during acquisition using an external reference (LockSpray) consisting of $0.2 \mathrm{ng} / \mathrm{mL}$ solution of leucine enkephalin infused at a flow rate of $20 \mu \mathrm{L} / \mathrm{min}$ via a LockSpray interface generating a reference ion at $554.2615 \mathrm{Da}\left([\mathrm{M}-\mathrm{H}]^{-}\right)$. All data collected in centroid mode were acquired using MassLynx V4.1 software (Waters Corporation, Milford, MA, USA).

\subsection{Principal Component Analysis (PCA) and Orthogonal} Partial Least Squares Discriminant Analysis (OPLS-DA). The original data peak detection of UPLC-TOF MS, principal component analysis (PCA), and orthogonal partial least squares discriminant analysis (OPLS-DA) were performed on all blood samples by using the MarkerLynx Application Manager in MassLynx V4.1 software. The quality window of peak detection was 0.05 , the retention time window was 0.2 , and the strength threshold was 50 . The Pareto method was used as the data standardization method for PCA and OPLS-DA. Then, we selected the signals from the S-plot figure of OPLS-DA which meet the following conditions: $X$-axis $>0.001$ and $Y$-axis $>0.8$ as the marker.

2.6. Identification of the Absorbed Components. We compared the retention time and mass data of the markers with the data of medicinal herbs and selected the data that matches. At the same time, we searched the original ingredients in the medicinal herbs in the mass spectrometry data of the plasma sample. Then, we analyzed the secondary mass spectrometry information of these data and identified their chemical structures based on the information provided by the fragment ions.

2.7. Cell Culture and MTS Colorimetric Assay. PC12 cells were cultured in DMEM (Jiangsu KeyGEN BioTECH Corp., Ltd; Nanjing, China) supplemented with 10\% FBS, at $37^{\circ} \mathrm{C}$ with $5 \% \mathrm{CO}_{2}$. PC12 cells in the logarithmic growth phase were seeded in a 384 -well plate $\left(3 \times 10^{4}\right.$ cells per well) and incubated at $37^{\circ} \mathrm{C}$ for $24 \mathrm{~h}$. Next, $100 \mu \mathrm{L}$ of the blank medium was added to the normal control group; $100 \mu \mathrm{L}$ of medium containing $30 \mu \mathrm{M} \mathrm{A} \beta_{25-35}$ was added to the model control group; $100 \mu \mathrm{L}$ of medium containing $30 \mu \mathrm{M} \mathrm{A} \beta_{25-35}$ and $250 \mu \mathrm{g} / \mathrm{mL}$ YZG extracting solution was added to YZG group. The galantamine hydrobromide group received $100 \mu \mathrm{L}$ of medium containing $30 \mu \mathrm{M} \mathrm{A} \beta_{25-}$ 35 and $30 \mu \mathrm{g} / \mathrm{mL}$ galantamine hydrobromide. The berberine hydrochloride group received $100 \mu \mathrm{L}$ medium containing $30 \mu \mathrm{M} \mathrm{A} \beta_{25-35}$ and $12.5 \mu \mathrm{g} / \mathrm{mL}$ berberine hydrochloride. The other groups treated with $\mathrm{A} \beta_{25-35}$ and part of the absorbed components were separately given $100 \mu \mathrm{L}$ of medium containing $30 \mu \mathrm{M} \mathrm{A} \beta_{25-35}$ and $25 \mu \mathrm{g} / \mathrm{mL}$ component. All groups were incubated for $48 \mathrm{~h}$. The volume fraction of DMSO in each group was not higher than $0.5 \%$, and 3 duplicate wells were set in each group. Subsequently, $10 \mu \mathrm{L}$ MTS $(1.90 \mathrm{mg} / \mathrm{mL})$ was added to each well and incubated at $37^{\circ} \mathrm{C}$ for $24 \mathrm{~h}$. The absorbance was measured at $480 \mathrm{~nm}$ using a spectrophotometer (Multimode Plate Reader EnVisionXcite; PerkinElme, Inc., Waltham, Massachusetts, USA). Cell viability was determined using the following equation: cell viability $(\%)=$ [OD $480 \mathrm{~nm}$ (drug)/OD $480 \mathrm{~nm}$ (control)] $\times 100 \%$. OD indicates optical density.

2.8. Statistical Analysis. All data are presented as the mean \pm standard error of the mean from at least three independent experiments. Data analysis was performed using GraphPad Prism 5.0 software (Graphpad Software, Inc., La Jolla, CA, USA). Statistical significance was also determined via a two-way analysis of variance.

\section{Results}

3.1. Optimization of UPLC/ESI-Q-TOF MS Conditions. To resolve the YZG components, the column type, temperature, and mobile phase were optimized. An ACQUITY UPLC BEH C18 column $(2.1 \mathrm{~mm} \times 100 \mathrm{~mm}, 1.7 \mu \mathrm{m}$, Waters Corporation, USA) was selected for the experiment. It offered a good resolution. To minimize peak width and maximize signal intensity, organic solvents including acetonitrile and methanol, several aqueous buffers, flow rate, and column temperature were investigated. Finally, optimal separation conditions were obtained. The MS conditions were optimized to maximize the response: capillary voltage, capillary temperature, collision energy, and gas flow. In negative ionization mode, all YZG analytes (extract and plasma) showed high sensitivity.

3.2. UPLC/ESI-Q-TOF MS Analysis of the Ingredients in YZG Samples and Cynomolgus Monkey Plasma. The total ion current for the YZG samples is shown in Figure 3. Seventy peaks were detected in YZG using the UPLC/ESI-Q-TOF MS technique; 63 compounds were structurally identified via comparison of retention time, MS, and MS/MS data of the reference compounds and those reported in the literature. The identified compounds are shown in Table 1. 


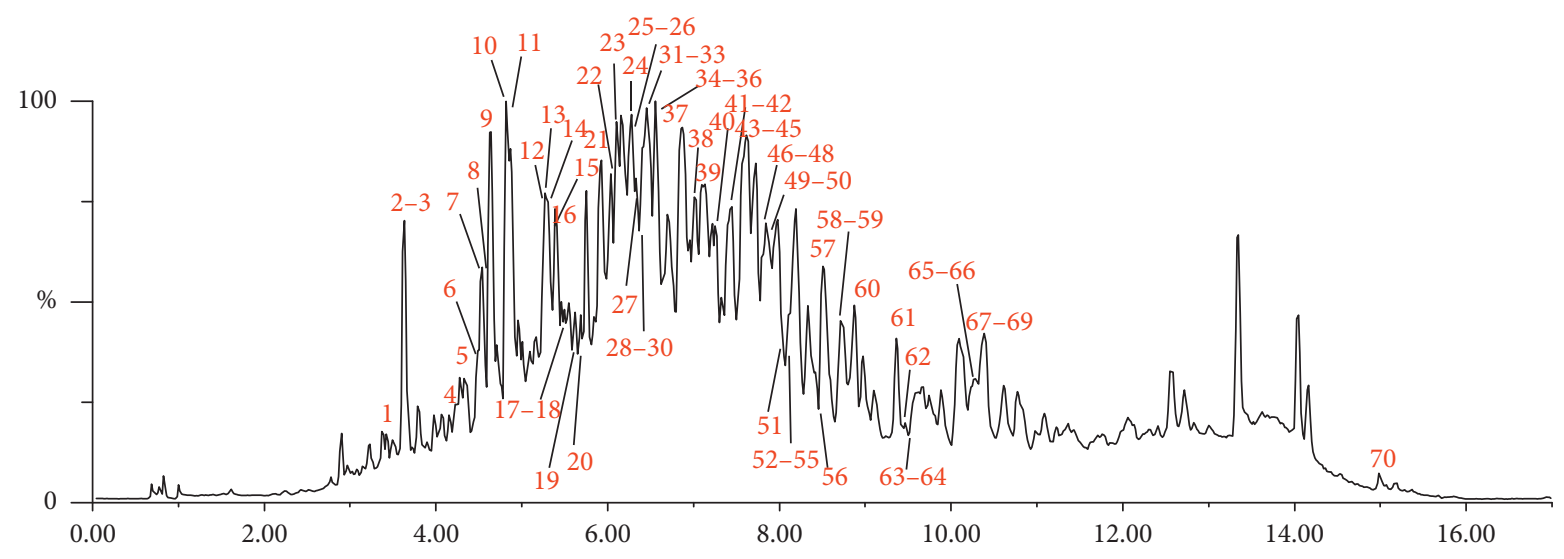

(a)

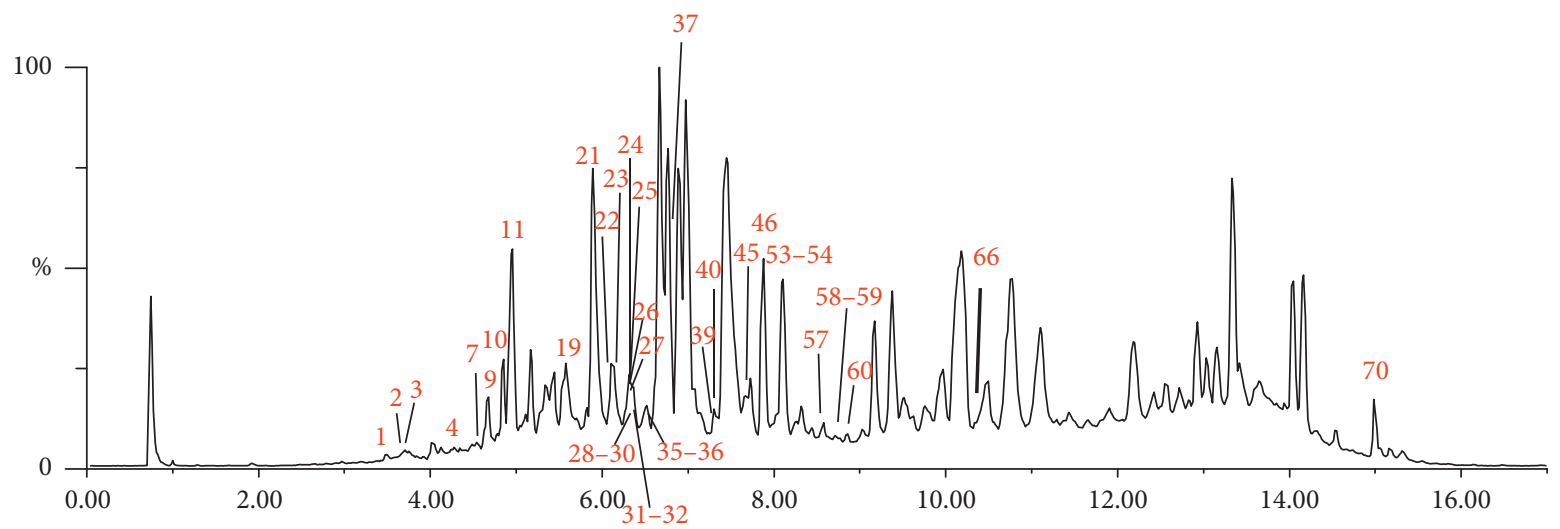

(b)

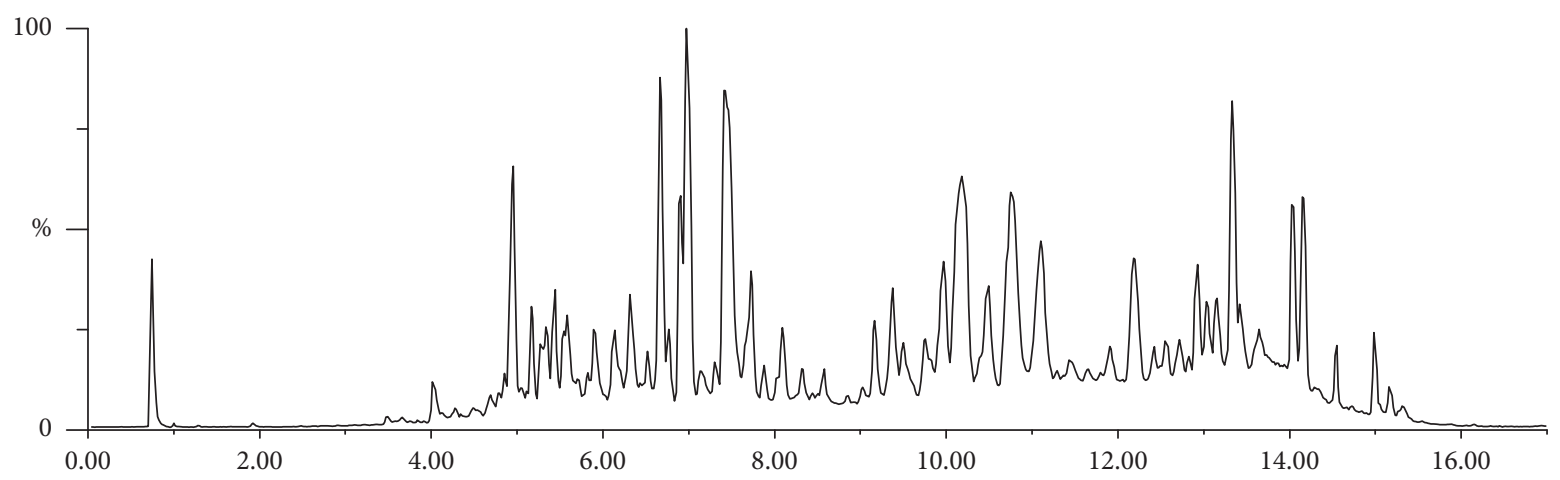

(c)

FIGURE 3: TIC chromatograms of YZG (a), the sample in cynomolgus monkeys' plasma after oral administration of YZG (b), and blank plasma in negative mode (c).

To identify the plasma components, the MS data of the drug plasma and blank plasma were compared using the MarkerLynx module in the MassLynx (V4.1) software.

The PCA scores of the UPLC/ESI-Q-TOF MS data of the drug plasma and the blank plasma were shown in Figure 4. It can be seen from the score chart that the scores of the drug plasma and the blank plasma differed significantly from each other in terms of the first principal component scores, showing significant differences in the data of the two groups and suggesting significant differences in the chemical components of the two groups.
The S diagram (S-plot) of OPLS-DA for the UPLC/ESIQ-TOF MS data of drug plasma and blank plasma is shown in Figure 5. Each point in the S-plot represents a data signal, the horizontal coordinate represents the contribution degree, the vertical coordinate represents the credibility, and the positive direction of the coordinate axis represents the signal of the drug group which is stronger than that of the blank group. The signal selected in the S-plot conforms to the following criteria: $X>0.001$ and $Y>0.8$.

36 compounds were found and structurally identified (Table 1). The MS/MS spectra and fragmentation pathways 
TABLE 1: Constituents identified from YZG by UPLC-ESI-Q-TOF-MS.

\begin{tabular}{|c|c|c|c|c|c|c|c|c|}
\hline No. & Rt & Formula & $\begin{array}{c}{\left[\mathrm{M}+\mathrm{HCOO}^{-}\right.} \\
(\mathrm{m} / z)\end{array}$ & $\begin{array}{c}{[\mathrm{M}-\mathrm{H}]^{-}} \\
(m / z)\end{array}$ & Fragment ions $(m / z)$ & CMP & Compound name & Reference \\
\hline 1 & 3.42 & $\mathrm{C}_{42} \mathrm{H}_{72} \mathrm{O}_{15}$ & 861.4843 & 815.4784 & $\begin{array}{c}653.4254,491.2235 \\
463.0880\end{array}$ & $\sqrt{ }$ & Notoginsenoside $\mathrm{M}$ & {$[23]$} \\
\hline 2 & 3.62 & $\mathrm{C}_{27} \mathrm{H}_{30} \mathrm{O}_{16}$ & & 609.1816 & 300.0555 & $\sqrt{ }$ & Rutin & {$[24]$} \\
\hline 3 & 3.72 & $\mathrm{C}_{8} \mathrm{H}_{8} \mathrm{O}_{5}$ & & 183.0308 & 124.0211 & $\sqrt{ }$ & Methyl gallate & {$[25]$} \\
\hline 4 & 4.22 & $\mathrm{C}_{48} \mathrm{H}_{82} \mathrm{O}_{19}$ & 1007.5439 & 961.5380 & $\begin{array}{l}997.5126,799.4825 \\
637.4305,475.1966\end{array}$ & $\sqrt{ }$ & $\begin{array}{c}\text { Notoginsenoside R3/R6/20-O- } \\
\text { Glucoginsenoside Rf }\end{array}$ & {$[23]$} \\
\hline 5 & 4.28 & $\mathrm{C}_{40} \mathrm{H}_{70} \mathrm{O}_{8}$ & 723.5082 & 677.5007 & $\begin{array}{c}740.4865,475.1963 \\
417.1550,284.0329\end{array}$ & $*$ & Unknown & \\
\hline 6 & 4.47 & $\mathrm{C}_{43} \mathrm{H}_{83} \mathrm{O}_{12}$ & 836.5914 & 790.5905 & 853.5441 & * & Unknown & \\
\hline 7 & 4.55 & $\mathrm{C}_{48} \mathrm{H}_{82} \mathrm{O}_{19}$ & 1007.5439 & 961.5406 & $\begin{array}{l}997.5145,799.4846 \\
637.4318,475.3790\end{array}$ & $\sqrt{ }$ & $\begin{array}{l}\text { Notoginsenoside R3/R6/20-O- } \\
\text { Glucoginsenoside Rf }\end{array}$ & {$[23]$} \\
\hline 8 & 4.57 & $\mathrm{C}_{32} \mathrm{H}_{38} \mathrm{O}_{15}$ & 707.2444 & 661.2957 & $\begin{array}{c}1323.5775,724.2191 \\
499.1783\end{array}$ & $*$ & Baohuoside A & $\Delta$ \\
\hline 9 & 4.61 & $\mathrm{C}_{47} \mathrm{H}_{80} \mathrm{O}_{18}$ & 977.5385 & 931.5317 & $\begin{array}{c}799.4850,637.4321 \\
475.3785\end{array}$ & $\sqrt{ }$ & Notoginsenoside R1 & {$[26]$} \\
\hline 10 & 4.81 & $\mathrm{C}_{48} \mathrm{H}_{82} \mathrm{O}_{18}$ & 991.5449 & 945.5477 & $\begin{array}{r}799.4883,637.4339 \\
619.4214,475.3792\end{array}$ & $\sqrt{ }$ & Ginsenoside $\mathrm{Re}$ & {$[27]$} \\
\hline 11 & 4.85 & $\mathrm{C}_{42} \mathrm{H}_{72} \mathrm{O}_{14}$ & 845.4936 & 799.4833 & $637.4339,475.3792$ & $\sqrt{ }$ & Ginsenoside Rg1 & {$[27]$} \\
\hline 12 & 5.24 & $\mathrm{C}_{39} \mathrm{H}_{50} \mathrm{O}_{20}$ & 883.2853 & 837.2800 & $\begin{array}{c}675.2280,529.2005 \\
513.1791,367.1183\end{array}$ & $*$ & Epimedin A1 & $\Delta$ \\
\hline 13 & 5.29 & $\mathrm{C}_{15} \mathrm{H}_{10} \mathrm{O}_{7}$ & & 301.0683 & $\begin{array}{c}273.0429,245.0488 \\
229.0532,179.0065 \\
151.0179,121.0346\end{array}$ & $*$ & Quercetin & $\Delta$ \\
\hline 14 & 5.31 & $\mathrm{C}_{38} \mathrm{H}_{48} \mathrm{O}_{19}$ & & 807.2960 & $\begin{array}{c}645.2173,513.1818,366.1090 \\
659.2647,513.1761\end{array}$ & $*$ & Epimedin B & $\Delta$ \\
\hline 15 & 5.38 & $\mathrm{C}_{38} \mathrm{H}_{50} \mathrm{O}_{19}$ & 867.2896 & 821.2642 & $\begin{array}{c}366.1201,351.0912 \\
323.0849,151.0094 \\
106.6611\end{array}$ & * & Epimedin $\mathrm{C}$ & $\Delta$ \\
\hline 16 & 5.40 & $\mathrm{C}_{38} \mathrm{H}_{50} \mathrm{O}_{19}$ & 867.3390 & 821.2833 & $659.2668,366.1226$ & * & Epimedin A & $\Delta$ \\
\hline 17 & 5.53 & $\mathrm{C}_{27} \mathrm{H}_{30} \mathrm{O}_{10}$ & & 513.1833 & $\begin{array}{c}367.1188,351.0876, \\
323.0917,151.0109,145.0312\end{array}$ & * & Isomer ${ }^{(47)}$ & $\Delta$ \\
\hline 18 & 5.53 & $\mathrm{C}_{33} \mathrm{H}_{40} \mathrm{O}_{15}$ & 721.2323 & 675.2302 & $529.1712,513.1846,367.1266$ & $*$ & Icariin & $\Delta$ \\
\hline 19 & 5.64 & $\mathrm{C}_{48} \mathrm{H}_{82} \mathrm{O}_{19}$ & 1007.5430 & 961.5372 & 799.4822, 637.3738 & $\sqrt{ }$ & Notoginsenoside N/R6 & {$[28]$} \\
\hline 20 & 5.67 & $\mathrm{C}_{54} \mathrm{H}_{92} \mathrm{O}_{23}$ & 1153.6027 & 1107.5963 & $\begin{array}{l}945.5418,799.4827 \\
637.4309,475.1394\end{array}$ & $*$ & Ginsenoside Re8 & {$[29]$} \\
\hline 21 & 5.90 & $\mathrm{C}_{59} \mathrm{H}_{100} \mathrm{O}_{27}$ & 1285.6840 & 1239.6516 & $\begin{array}{c}\text { 1107.5920, } 945.5372 \\
783.4835,621.4189 \\
459.3343\end{array}$ & $\sqrt{ }$ & Notoginsenoside Fa & $\Delta$ \\
\hline 22 & 6.09 & $\mathrm{C}_{42} \mathrm{H}_{72} \mathrm{O}_{14}$ & 845.4875 & 799.4935 & $\begin{array}{c}637.4294,619.5074 \\
475.3772\end{array}$ & $\sqrt{ }$ & Ginsenoside Rf & {$[28]$} \\
\hline 23 & 6.11 & $\mathrm{C}_{54} \mathrm{H}_{92} \mathrm{O}_{23}$ & 1153.6823 & 1107.6227 & $\begin{array}{l}945.5391,783.4855 \\
621.4235,459.3650\end{array}$ & $\sqrt{ }$ & Ginsenoside Rb1 & {$[30]$} \\
\hline 24 & 6.21 & $\mathrm{C}_{52} \mathrm{H}_{86} \mathrm{O}_{21}$ & 1091.5645 & 1045.7167 & $\begin{array}{l}913.5291,751.4626 \\
605.4065,473.3609\end{array}$ & $\sqrt{ }$ & Gypenoside XLIX & $\Delta$ \\
\hline 25 & 6.26 & $\mathrm{C}_{53} \mathrm{H}_{90} \mathrm{O}_{22}$ & 1123.5693 & 1077.6210 & $945.5375,783.4875$ & $\sqrt{ }$ & Ginsenoside Rc & {$[30]$} \\
\hline 26 & 6.28 & $\mathrm{C}_{41} \mathrm{H}_{70} \mathrm{O}_{13}$ & 815.4929 & 769.4709 & $\begin{array}{c}637.4373,619.4420 \\
475.3855\end{array}$ & $\sqrt{ }$ & 20(S)-Notoginsenoside R2 & $\Delta$ \\
\hline 27 & 6.33 & $\mathrm{C}_{41} \mathrm{H}_{70} \mathrm{O}_{13}$ & 815.4812 & 769.4928 & $\begin{array}{c}637.4339,475.3815 \\
1209.6248,1077.5825\end{array}$ & $\sqrt{ }$ & Notoginsenoside R2/F3/F5 & {$[28]$} \\
\hline 28 & 6.40 & $\mathrm{C}_{63} \mathrm{H}_{106} \mathrm{O}_{30}$ & & 1341.5354 & $\begin{array}{l}945.5411,783.4882 \\
621.4359,459.3843\end{array}$ & $\sqrt{ }$ & Notoginsenoside Q & {$[28]$} \\
\hline 29 & 6.41 & $\mathrm{C}_{48} \mathrm{H}_{76} \mathrm{O}_{19}$ & & 955.4913 & $793.4395,569.3852$ & $\sqrt{ }$ & Ginsenoside rRo & {$[31]$} \\
\hline 30 & 6.42 & $\mathrm{C}_{53} \mathrm{H}_{90} \mathrm{O}_{22}$ & 1123.5911 & 1077.5951 & $\begin{array}{l}945.5395,783.4940 \\
621.4150,459.3434\end{array}$ & $\sqrt{ }$ & Ginsenoside Rb2 & {$[28]$} \\
\hline 31 & 6.44 & $\mathrm{C}_{53} \mathrm{H}_{90} \mathrm{O}_{22}$ & 1123.5911 & 1077.5911 & $\begin{array}{c}945.5429,783.4907 \\
459.3839\end{array}$ & $\sqrt{ }$ & Ginsenoside Rb3 & {$[28]$} \\
\hline 32 & 6.46 & $\mathrm{C}_{42} \mathrm{H}_{72} \mathrm{O}_{13}$ & 829.5134 & 783.5062 & $\begin{array}{c}637.4319,619.4190 \\
475.3806\end{array}$ & $\sqrt{ }$ & 20(S)-Ginsenoside Rg2 & {$[30]$} \\
\hline
\end{tabular}


TABle 1: Continued.

\begin{tabular}{|c|c|c|c|c|c|c|c|c|}
\hline No. & Rt & Formula & $\begin{array}{c}{\left[\mathrm{M}+\underset{\mathrm{HCOO}]^{-}}{(m / z)}\right.} \\
\end{array}$ & $\begin{array}{c}{[\mathrm{M}-\mathrm{H}]^{-}} \\
(m / z)\end{array}$ & Fragment ions $(\mathrm{m} / \mathrm{z})$ & CMP & Compound name & Reference \\
\hline 33 & 6.50 & $\mathrm{C}_{41} \mathrm{H}_{70} \mathrm{O}_{13}$ & 815.4929 & 769.4709 & $\begin{array}{c}637.4373,619.4420 \\
475.3855\end{array}$ & $*$ & 20(R)-Notoginsenoside R2 & $\Delta$ \\
\hline 34 & 6.56 & $\mathrm{C}_{36} \mathrm{H}_{62} \mathrm{O}_{9}$ & 683.4399 & 637.4317 & 475.3788 & $*$ & $\begin{array}{l}\text { 20(S)-Ginsenoside Rh1/20(R)- } \\
\text { Ginsenoside Rh1 }\end{array}$ & {$[28]$} \\
\hline 35 & 6.56 & $\mathrm{C}_{42} \mathrm{H}_{72} \mathrm{O}_{13}$ & 829.4958 & 783.4899 & $\begin{array}{c}637.4317,475.3788 \\
1107.5959,945.5424\end{array}$ & $\sqrt{ }$ & 20(R)-Ginsenoside Rg2 & {$[30]$} \\
\hline 36 & 6.59 & $\mathrm{C}_{56} \mathrm{H}_{94} \mathrm{O}_{24}$ & 1195.6116 & 1149.6077 & $\begin{array}{c}\text { 783.4896, } 637.4297 \\
475.3786\end{array}$ & $\sqrt{ }$ & Yesanchinoside F & {$[32]$} \\
\hline 37 & 6.85 & $\mathrm{C}_{48} \mathrm{H}_{82} \mathrm{O}_{18}$ & 991.5511 & 945.5786 & $\begin{array}{c}783.4921,621.4363 \\
459.3667\end{array}$ & $\sqrt{ }$ & Ginsenoside Rd & {$[28]$} \\
\hline 38 & 6.98 & $\mathrm{C}_{51} \mathrm{H}_{100} \mathrm{O}_{30}$ & 1237.6125 & 1191.6177 & $\begin{array}{c}1029.5610,915.5285 \\
637.4301,475.3689\end{array}$ & * & Unknown & \\
\hline 39 & 7.21 & $\mathrm{C}_{52} \mathrm{H}_{86} \mathrm{O}_{21}$ & 943.5059 & 897.5676 & $\begin{array}{c}765.4807,751.4272 \\
681.4173,619.3864 \\
487.3429\end{array}$ & $\sqrt{ }$ & Gypenoside A & $\Delta$ \\
\hline 40 & 7.26 & $\mathrm{C}_{55} \mathrm{H}_{92} \mathrm{O}_{23}$ & 1165.6019 & 1119.5986 & $\begin{array}{c}783.4898,621.4372 \\
459.3844\end{array}$ & $\sqrt{ }$ & Ginsenoside Rs2 & {$[28]$} \\
\hline 41 & 7.44 & $\mathrm{C}_{27} \mathrm{H}_{30} \mathrm{O}_{11}$ & 575.3054 & 529.1713 & $\begin{array}{c}\text { 1105.6187, } 1059.6130 \\
367.1176\end{array}$ & $*$ & Icariside I & \\
\hline 42 & 7.49 & $\mathrm{C}_{47} \mathrm{H}_{80} \mathrm{O}_{17}$ & 961.5392 & 915.5334 & $783.4893,621.4371$ & $*$ & $\begin{array}{c}\text { Notoginsenoside Fe/ } \\
\text { gynosaponin I/vinaginsenoside } \\
\text { R16/R17 }\end{array}$ & {$[28]$} \\
\hline 43 & 7.61 & $\mathrm{C}_{60} \mathrm{H}_{116} \mathrm{O}_{36}$ & 1457.7361 & 1411.7128 & $\begin{array}{c}1265.6560,1133.6172 \\
987.5623,841.4951 \\
475.3750\end{array}$ & $*$ & Unknown & \\
\hline 44 & 7.62 & $\mathrm{C}_{48} \mathrm{H}_{82} \mathrm{O}_{18}$ & 991.5460 & 945.5498 & $\begin{array}{c}799.4841,637.4338 \\
619.4188,475.3750\end{array}$ & $*$ & Unknown & \\
\hline 45 & 7.63 & $\mathrm{C}_{51} \mathrm{H}_{86} \mathrm{O}_{21}$ & 1033.6028 & 987.5575 & $\begin{array}{c}945.5498,927.5349 \\
841.4951\end{array}$ & $\sqrt{ }$ & Pseudoginsenoside Rc1 & {$[28]$} \\
\hline 46 & 7.81 & $\mathrm{C}_{55} \mathrm{H}_{92} \mathrm{O}_{23}$ & 1165.6125 & 1119.6310 & $\begin{array}{c}\text { 1077.5851, 1059.5736, } \\
945.5401,927.5275 \\
765.4456,621.3965 \\
459.2525\end{array}$ & $\sqrt{ }$ & Ginsenoside Rs1 & {$[28]$} \\
\hline 47 & 7.82 & $\mathrm{C}_{27} \mathrm{H}_{30} \mathrm{O}_{10}$ & & 513.1867 & $\begin{array}{c}366.1149,351.0916 \\
323.0886,151.0092 \\
132.0236\end{array}$ & $*$ & Baohuoside I & $\Delta$ \\
\hline 48 & 7.82 & $\mathrm{C}_{50} \mathrm{H}_{98} \mathrm{O}_{29}$ & 1207.6355 & 1161.6063 & $1175.6207,1161.6063$ & $*$ & Unknown & \\
\hline 49 & 7.85 & $\mathrm{C}_{41} \mathrm{H}_{70} \mathrm{O}_{14}$ & 831.4783 & 785.4698 & 653.4258 & $*$ & Notoginsenoside Rw2 & {$[33]$} \\
\hline 50 & 7.89 & $\mathrm{C}_{54} \mathrm{H}_{86} \mathrm{O}_{24}$ & 1163.6464 & 1117.6188 & $\begin{array}{c}\text { 1057.5961, 971.5587 } \\
929.5478\end{array}$ & $*$ & $\begin{array}{c}\text { Hemsloside G2/ginsenoside } \\
\text { ROA }\end{array}$ & {$[34]$} \\
\hline 51 & 8.05 & $\mathrm{C}_{42} \mathrm{H}_{70} \mathrm{O}_{12}$ & 811.4849 & 765.4782 & $619.4163,457.2605$ & $*$ & Ginsenoside Rg6 & $\Delta$ \\
\hline 52 & 8.14 & $\mathrm{C}_{52} \mathrm{H}_{104} \mathrm{O}_{30}$ & 1253.6549 & 1207.6088 & $\begin{array}{c}1207.6088,1075.5676 \\
943.5283\end{array}$ & $*$ & Unknown & \\
\hline 53 & 8.16 & $\mathrm{C}_{42} \mathrm{H}_{72} \mathrm{O}_{13}$ & 829.4985 & 783.4911 & $\begin{array}{l}637.4318,475.3790 \\
945.5425927 .5313\end{array}$ & $\sqrt{ }$ & Ginsenoside Fc & {$[28]$} \\
\hline 54 & 8.17 & $\mathrm{C}_{51} \mathrm{H}_{86} \mathrm{O}_{21}$ & 1033.5596 & 987.5541 & $\begin{array}{l}783.4911,765.4796 \\
621.4370,459.3835\end{array}$ & $\sqrt{ }$ & Quinquenoside III & {$[28]$} \\
\hline 55 & 8.18 & $\mathrm{C}_{42} \mathrm{H}_{72} \mathrm{O}_{14}$ & 845.4895 & 799.4831 & $\begin{array}{c}637.4308,619.4199 \\
475.3775\end{array}$ & $*$ & Isomer $^{(22)}$ & {$[28]$} \\
\hline 56 & 8.42 & $\mathrm{C}_{48} \mathrm{H}_{82} \mathrm{O}_{16}$ & 959.5573 & 913.5543 & $\begin{array}{l}767.4940,621.4166 \\
475.3465,146.9997\end{array}$ & $*$ & Gynosaponin II & {$[35]$} \\
\hline 57 & 8.50 & $\mathrm{C}_{56} \mathrm{H}_{94} \mathrm{O}_{24}$ & & 1149.6082 & $\begin{array}{c}1107.7029,945.5416 \\
927.5334,783.4859 \\
765.3539,621.4349 \\
459.3838\end{array}$ & $\sqrt{ }$ & Quinquenoside R1 & {$[28]$} \\
\hline 58 & 8.72 & $\mathrm{C}_{42} \mathrm{H}_{72} \mathrm{O}_{13}$ & 829.4963 & 783.4920 & $\begin{array}{c}767.4591,621.4376 \\
459.3846\end{array}$ & $\sqrt{ }$ & 20(S)-Ginsenoside Rg3 & {$[23]$} \\
\hline 59 & 8.77 & $\mathrm{C}_{42} \mathrm{H}_{72} \mathrm{O}_{13}$ & 829.4974 & 783.4915 & $\begin{array}{c}765.4796,621.4368 \\
459.3842\end{array}$ & $\sqrt{ }$ & Isomer $^{(58)}$ & {$[23]$} \\
\hline
\end{tabular}


TABLE 1: Continued.

\begin{tabular}{|c|c|c|c|c|c|c|c|c|}
\hline No. & Rt & Formula & $\begin{array}{c}{\left[\mathrm{M}+\mathrm{HCOO}^{-}\right.} \\
(m / z) \\
\end{array}$ & $\begin{array}{c}{[\mathrm{M}-\mathrm{H}]^{-}} \\
(m / z)\end{array}$ & Fragment ions $(\mathrm{m} / \mathrm{z})$ & CMP & Compound name & Reference \\
\hline 60 & 8.86 & $\mathrm{C}_{42} \mathrm{H}_{72} \mathrm{O}_{13}$ & 829.5020 & 783.4990 & $\begin{array}{c}621.4348,459.3762 \\
161.0500\end{array}$ & $\sqrt{ }$ & 20(R)-Ginsenoside Rg3 & {$[23]$} \\
\hline 61 & 9.39 & $\mathrm{C}_{36} \mathrm{H}_{62} \mathrm{O}_{9}$ & 683.4375 & 637.4319 & 475.3790 & $*$ & $\begin{array}{l}\text { 20(S)-Ginsenoside } \mathrm{Rh} 1 / 20(\mathrm{R}) \text { - } \\
\text { Ginsenoside } \mathrm{Rh} 1\end{array}$ & {$[28]$} \\
\hline 62 & 9.54 & $\mathrm{C}_{47} \mathrm{H}_{80} \mathrm{O}_{18}$ & 977.4802 & 931.5010 & $\begin{array}{l}799.4818,769.4988 \\
751.4977,637.3975\end{array}$ & $*$ & Isomer ${ }^{(9)}$ & {$[28]$} \\
\hline 63 & 9.59 & $\mathrm{C}_{49} \mathrm{H}_{80} \mathrm{O}_{18}$ & 1001.5699 & 955.5613 & $913.5525,767.4947$ & $*$ & Gylongiposide I & {$[36]$} \\
\hline 64 & 9.60 & $\mathrm{C}_{47} \mathrm{H}_{80} \mathrm{O}_{17}$ & 961.5573 & 915.5556 & $\begin{array}{c}783.4860,621.4335 \\
459.3809\end{array}$ & $*$ & $\begin{array}{c}\text { Notoginsenoside Fe/ } \\
\text { gynosaponin I/vinaginsenoside } \\
\text { R16/R17 }\end{array}$ & {$[28]$} \\
\hline 65 & 10.22 & $\mathrm{C}_{42} \mathrm{H}_{70} \mathrm{O}_{12}$ & 811.4861 & 765.4803 & $\begin{array}{c}619.4220,603.4269 \\
457.2275\end{array}$ & $*$ & Ginsenoside Rg6 & {$[28]$} \\
\hline 66 & 10.26 & $\mathrm{C}_{42} \mathrm{H}_{72} \mathrm{O}_{13}$ & 829.4875 & 783.4943 & $621.4368,459.3837$ & $\sqrt{ }$ & Ginsenoside F2 & {$[28]$} \\
\hline 67 & 10.36 & $\mathrm{C}_{42} \mathrm{H}_{70} \mathrm{O}_{12}$ & 811.4857 & 765.4805 & $\begin{array}{c}619.4229,603.4269 \\
457.2340\end{array}$ & $*$ & Ginsenoside F4 & {$[28]$} \\
\hline 68 & 10.38 & $\mathrm{C}_{42} \mathrm{H}_{70} \mathrm{O}_{12}$ & 811.4849 & 765.4792 & 603.3373 & * & Ginsenoside Rg5 & {$[28]$} \\
\hline 69 & 10.40 & $\mathrm{C}_{42} \mathrm{H}_{70} \mathrm{O}_{12}$ & 811.4850 & 765.4794 & $603.3384,483.2727$ & $*$ & Ginsenoside Rk1 & {$[28]$} \\
\hline 70 & 15.05 & $\mathrm{C}_{47} \mathrm{H}_{80} \mathrm{O}_{17}$ & 961.6223 & 915.5983 & $\begin{array}{l}978.5970,783.5145 \\
621.3875,459.3448\end{array}$ & $\sqrt{ }$ & $\begin{array}{c}\text { Notoginsenoside Fe/ } \\
\text { gynosaponin I/vinaginsenoside } \\
\text { R16/R17 }\end{array}$ & {$[28]$} \\
\hline
\end{tabular}

Abbreviations: Rt, retention time; CMP, cynomolgus monkeys plasma; Ref., references. $\sqrt{ }:$ compound detected; $^{*}:$ compound not detected. isomer $^{\left({ }^{\circ} \circ\right)}$ : numbers in parentheses represent the peak corresponding to isomers; $\Delta$ : the data are consistent with the standard substance.

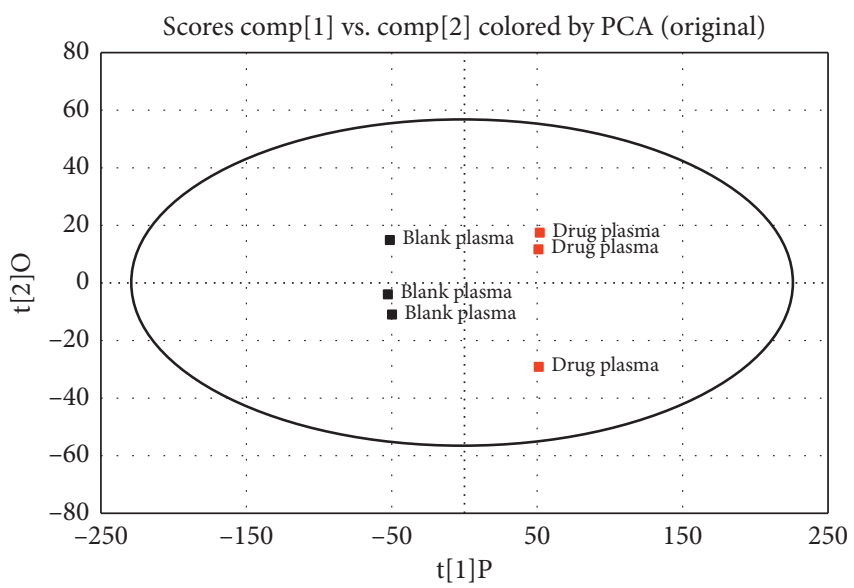

FIGURE 4: Score plot from principal component analysis (PCA) of drug plasma and the blank plasma.

of some absorbed components in plasma are shown in Figures 6-9.

Peak 9 (Rt $4.61 \mathrm{~min}$ ) produced $[\mathrm{M}-\mathrm{H}]^{-}$ions at $\mathrm{m} / z$ 931.5317 and $[\mathrm{M}+\mathrm{HCOO}]^{-}$ions at $\mathrm{m} / z 977.5385$ indicating that the molecular formula was C47H80O18. Its MS/MS data showed characteristic fragments formed at $\mathrm{m} / z$ 799.4850 and 637.4321 as shown in Figure 6. This suggests the loss of one xylosyl group followed by one glycosyl group. The mass fragmentation behavior of this compound suggested that it was notoginsenoside R1, which was confirmed by comparison to the literature [26].

Peak 23 (RT $6.11 \mathrm{~min}$ ) produced $[\mathrm{M}-\mathrm{H}]^{-}$ions at $\mathrm{m} / z$ 1107.6023 indicating that the molecular formula was $\mathrm{C}_{54} \mathrm{H}_{92} \mathrm{O}_{23}$. The MS/MS data $m / z 945.5431$ (Figure 7)

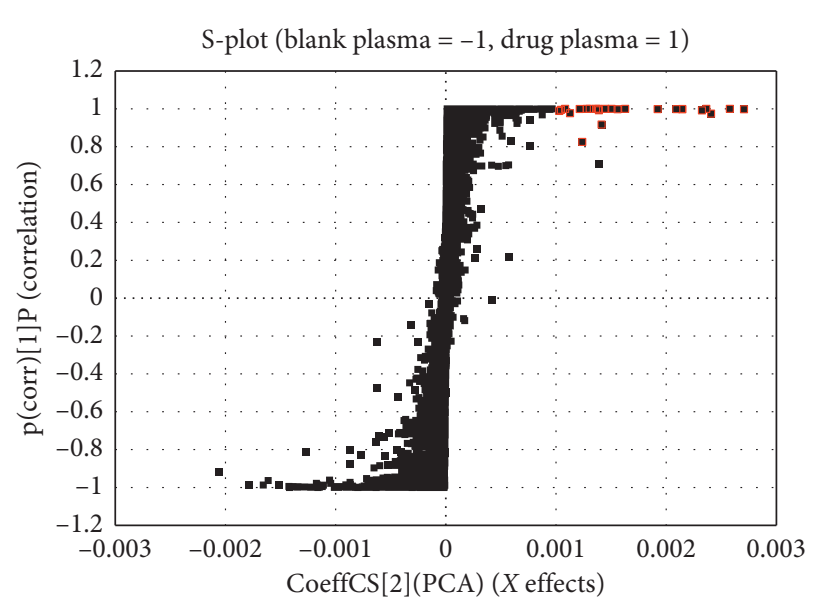

FIGURE 5: S-plot from orthogonal partial least squares discriminant analysis (OPLS-DA) of drug plasma and the blank plasma.

suggests the loss of one glycosidic group and $m / z 783.4896$ suggests one more glycosidic group loss. In addition, the MS/MS data indicated the loss of another two glycosidic groups, which are shown at $m / z 621.4235$ and $m / z 459.3650$, respectively. The mass fragmentation behavior of this compound suggested that it could be identified as ginsenoside $\mathrm{Rb} 1$ as confirmed by comparison to the reported data.

Peak 24 (Rt $6.21 \mathrm{~min}$ ) produced a significant $[\mathrm{M}-\mathrm{H}]^{-}$ion at $\mathrm{m} / z 1045.7167$ and $[\mathrm{M}+\mathrm{HCOO}]^{-}$ions at $\mathrm{m} / z 1091.5645$. The molecular formula $\left(\mathrm{C}_{52} \mathrm{H}_{86} \mathrm{O}_{21}\right)$ could be deduced via elemental composition. The characteristic fragments formed were at $m / z$ 913.5291, 751.4626, 605.4065, and 473.3609, which were consistent with the standard compound. Thus, peak 24 was identified as gypenoside XLIX, and its fragmentation pathway is shown in Figure 8. 


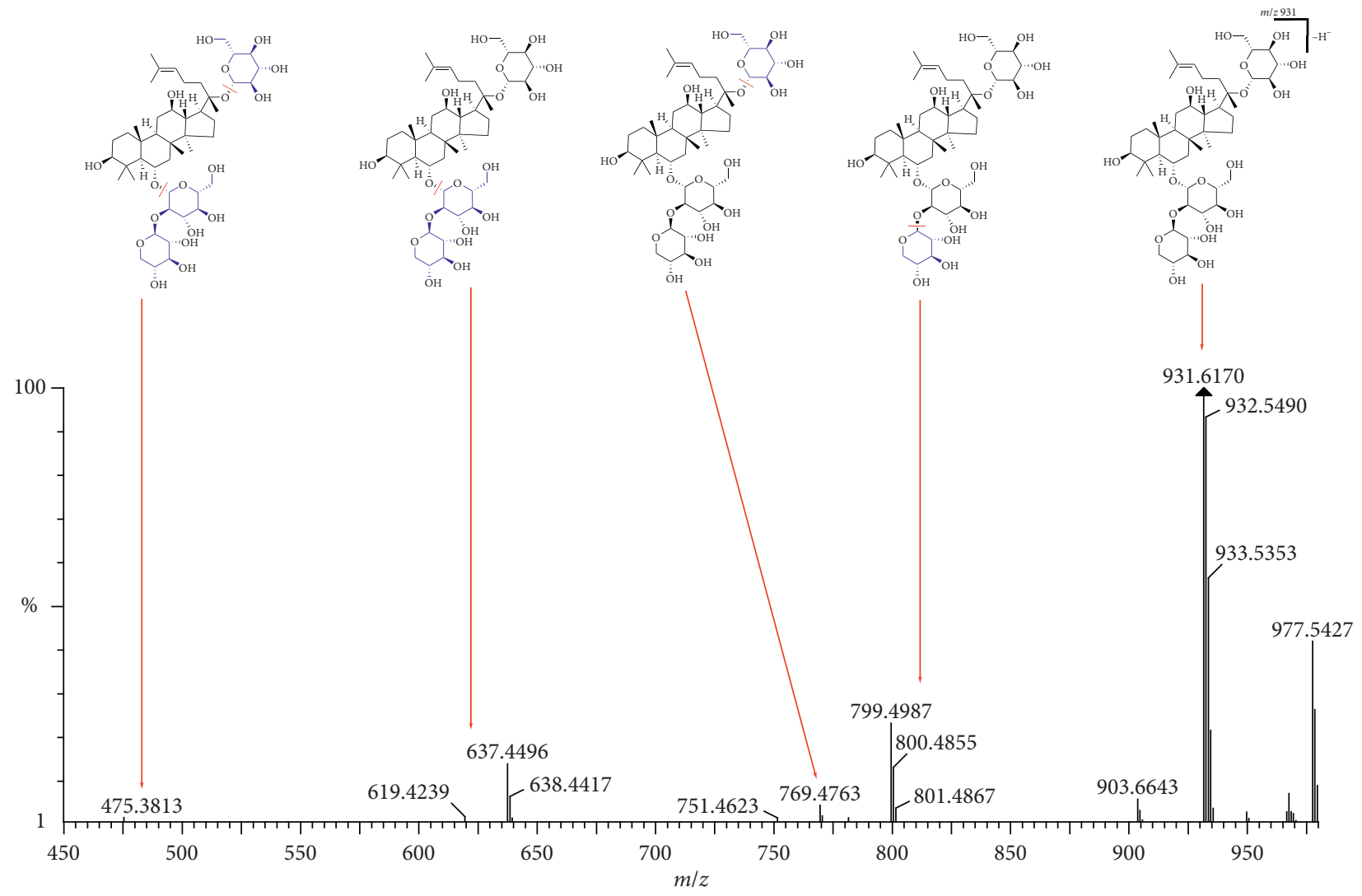

Figure 6: LC-MS spectrum and fragmentation pathway of notoginsenoside R1.

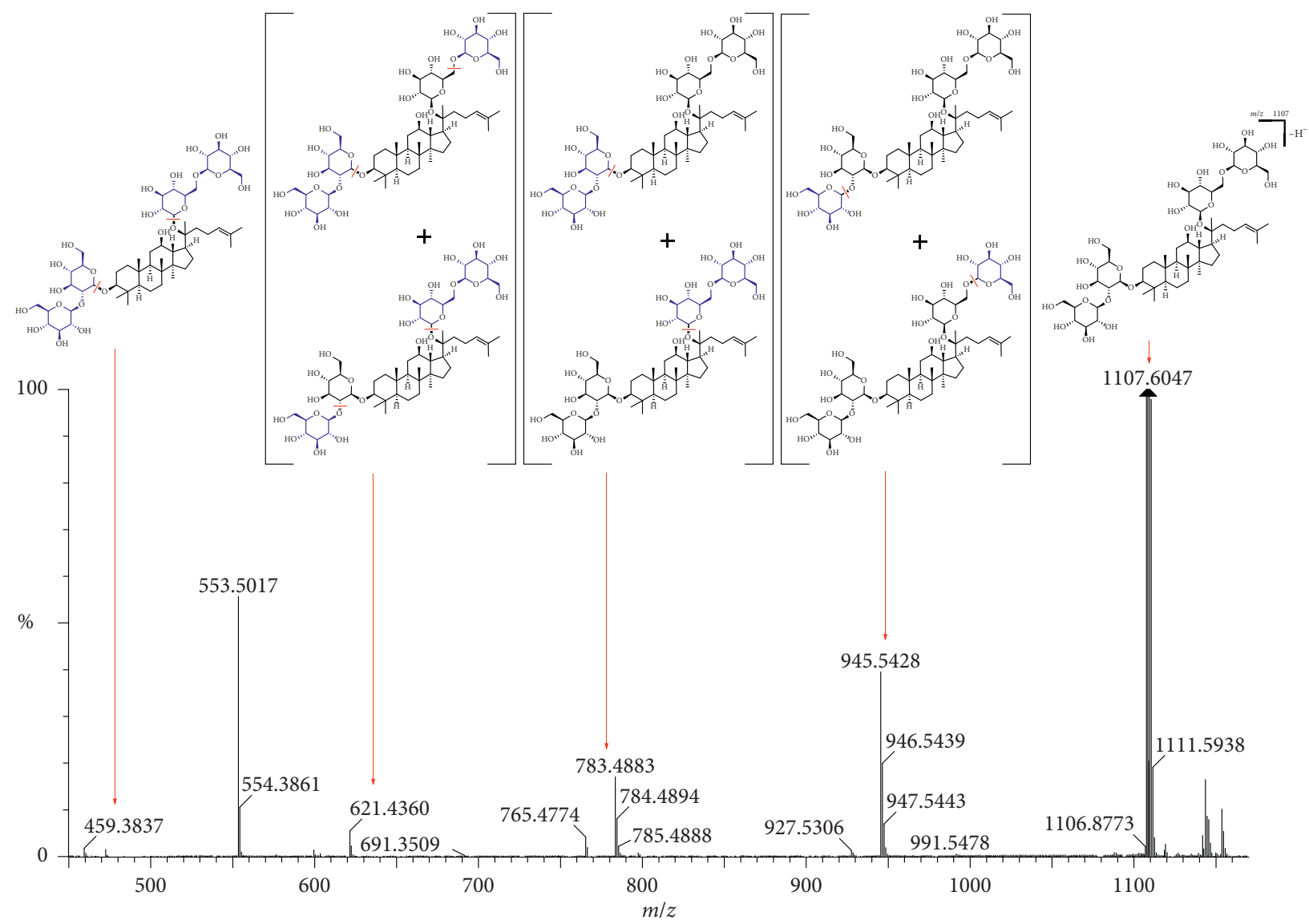

FIGURE 7: LC-MS spectrum and fragmentation pathway of ginsenoside Rb1. 


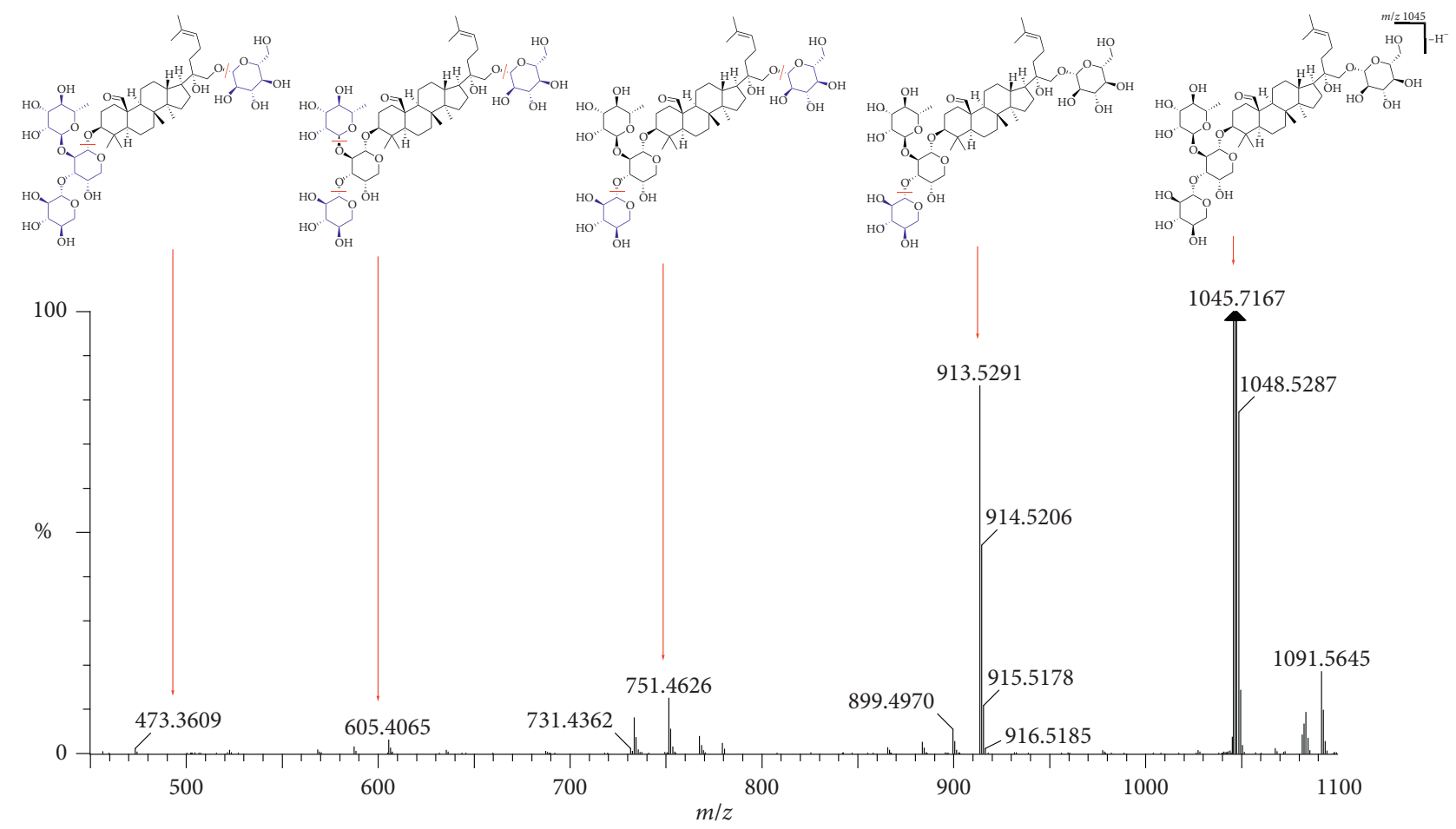

FIGURE 8: LC-MS spectrum and fragmentation pathway of gypenoside XLIX.

Peak 39 (RT $7.21 \mathrm{~min}$ ) produced the $[\mathrm{M}-\mathrm{H}]^{-}$ion at $\mathrm{m} / \mathrm{z}$ 897.5676 and $[\mathrm{M}+\mathrm{HCOO}]^{-}$ion at $\mathrm{m} / z 943.5059$ indicating that the molecular formula was $\mathrm{C}_{46} \mathrm{H}_{74} \mathrm{O}_{17}$. The characteristic fragments formed were at $\mathrm{m} / z$ 765.4807, 751.4272, 681.4173, 619.3864, and 487.3429. The fragments at $\mathrm{m} / z$ 765.4807 and 751.4272 indicated the loss of one xylosyl group and one rhamnosyl group, respectively; the characteristic fragments at $m / z 681.4173$ indicated the loss of both. The fragmentation pathway of peak 39 is shown in Figure 9.

3.3. Pharmacological Action of Absorbed Components. The pathological features of Alzheimer's disease mainly include senile plaque (SP) formed by the deposition of $\beta$-amyloid $(\mathrm{A} \beta)$ outside the neurons as well as neurofibrillary tangles formed by hyperphosphorylation of tau protein in neurons, neufibrillary tangles (NFTs), and neuronal loss [17]. Excessive deposition of $\mathrm{A} \beta$ can induce oxidative stress; result in excessive accumulation of free radicals; lead to peroxidative damage of biomacromolecules lipids, proteins, DNA, and RNA [37]; and cause neuronal apoptosis [38]. Therefore, $A \beta$ induced oxidative stress plays a vital role in the pathogenesis of $\mathrm{AD}[38,39]$. Neuroinflammation is also one of the pathological features of $\mathrm{AD}[40]$ and is an essential mediator of $\mathrm{A} \beta$-induced neuronal death and another essential factor in the induction of $\mathrm{AD}$ pathology in addition to oxidative stress [41]. There is increasing evidence that $\mathrm{A} \beta$-induced inflammatory responses are an essential component of $\mathrm{A} \beta$ neurotoxicity [42]. PC12 cells are clonal cell lines of rat adrenal chromaffin cells. They are neurogenic and have typical neuroendocrine cell characteristics. They are widely used in the study of neuronal differentiation, ion channels, receptors, and transmitter secretion. They are also one of the most common cell lines for studying neurotoxicity and are useful in vitro cell models [43]. Therefore, the active fragment $\mathrm{A} \beta_{23-35}$ was used in this experiment to induce PC12 cells to establish a neuronal injury model.

After treating with $\mathrm{A} \beta_{25-35}$, the PC12 cells in the model control group were obviously damaged - the cell viability of the model control group obviously decreased (compared with the blank control group, $p<0.05$ ). Compared with the model control group, the positive control drugs galantamine and berberine could obviously protect PC12 cells from the damage of $\mathrm{A} \beta_{25-35}(p<0.05)$. Ginsenoside $\mathrm{Rb} 1$, ginsenoside $\mathrm{Rb} 2$, ginsenoside $\mathrm{Rd}$, ginsenoside $\mathrm{Re}$, notoginsenoside $\mathrm{Fa}$, notoginsenoside R1, notoginsenoside R2, gypenoside A, and gypenoside XLIX have apparent protective effects on PC12 cells from the damage of $\mathrm{A} \beta_{25-35}(p<0.05)$ as shown in Figure 10. Table 1 shows that 36 compounds of YZG were absorbed in cynomolgus monkey plasma; however, only 14 of these compounds were used for pharmacological experiments into the neuroprotection effects. The other absorbed compounds in cynomolgus monkey plasma will be separated or purchased for the pharmacodynamic screening of neuroprotection effects. The active components in Figure 10 will be further verified in zebrafish or mouse models.

\section{Discussion}

YZG is a health food containing six TCMs. We recently showed that YZG improved memory barriers in animal experiments and $\mathrm{YZG}$ could protect the $\mathrm{PC} 12$ from the damage induced by protein $\mathrm{A} \beta_{25-35}$ [12-15]. The related effects of these six herbs in YZG have also been reported. 


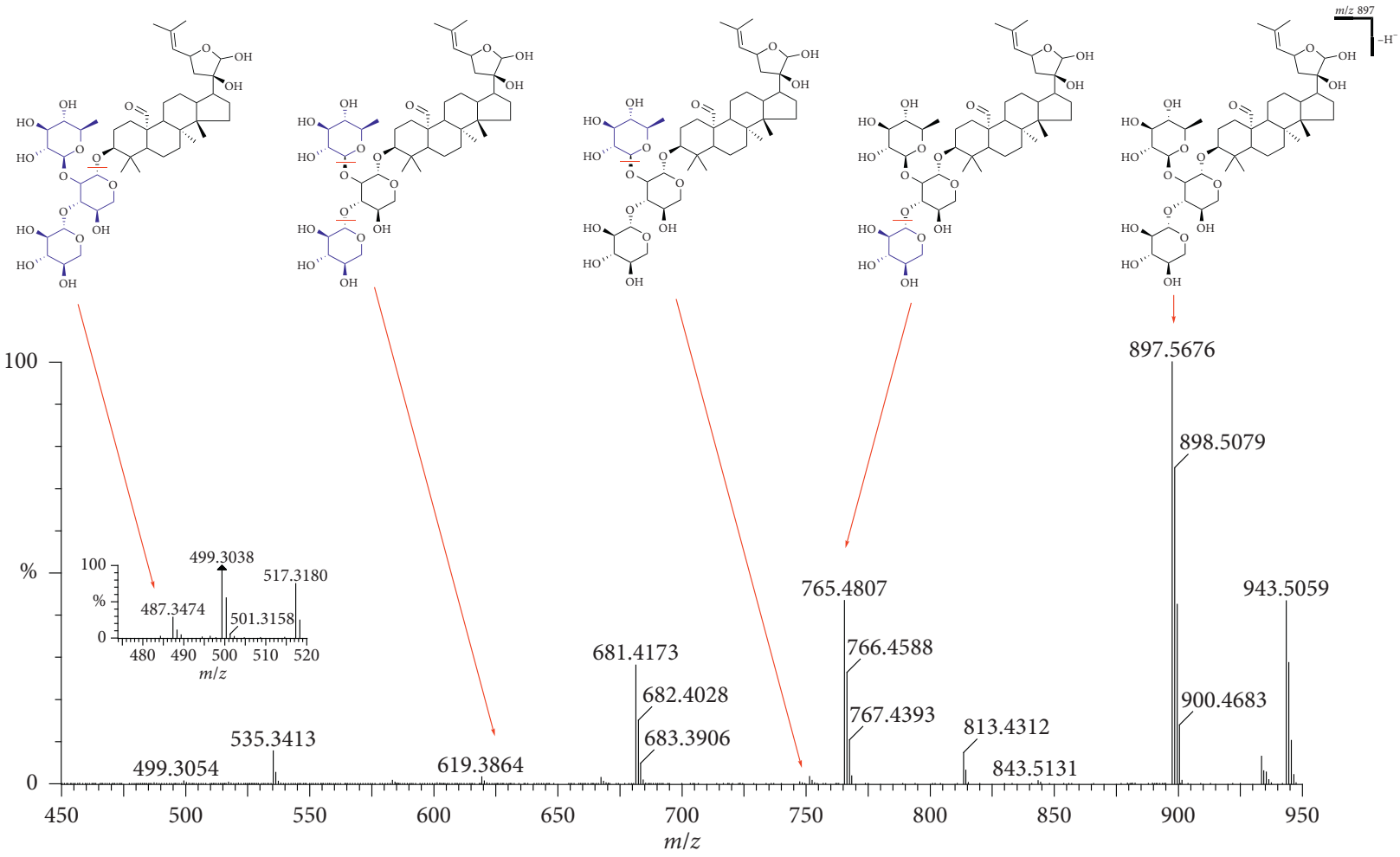

FIGURE 9: LC-MS spectrum and fragmentation pathway of gypenoside A.

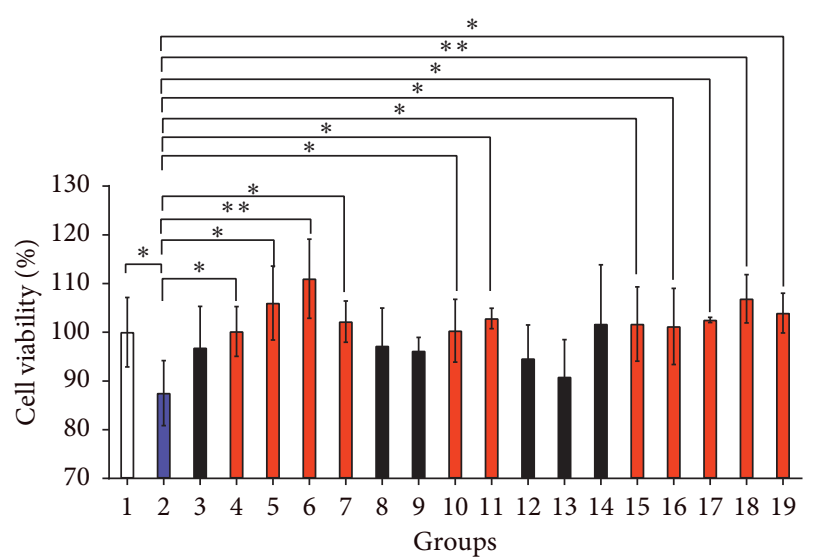

FIGURE 10: Screening of the components with a protective effect on PC12 treated with A $\beta 25-35$. Samples are as follows: 1, blank control group; 2, model control group; 3, YZG group; 4, galantamine hydrobromide group; 5, berberine hydrochloride group; 6, ginsenoside $\mathrm{Rb} 1$ group; 7, ginsenoside $\mathrm{Rb} 2$ group; 8 , ginsenoside $\mathrm{Rb} 3$ group; 9, ginsenoside Rc group; 10, ginsenoside Rd group; 11, ginsenoside Re group; 12, ginsenoside Rf group; 13, ginsenoside Rg1 group; 14, ginsenoside $\mathrm{Rg} 2$ group; 15, notoginsenoside $\mathrm{Fa}$ group; 16, notoginsenoside R1 group; 17, notoginsenoside R2 group; 18, gypenoside A group; and 19, gypenoside XLIX group.

Ginsenosides can reduce the formation of amyloid $\beta$-protein $(\mathrm{A} \beta)$ through inhibiting $\beta$ - and $\gamma$-secretase activity or by activating the nonamyloidogenic pathway, inhibit acetylcholinesterase activity and induced neurotoxicity, and reduce the generation of reactive oxygen species and neuroinflammatory response by $\mathrm{A} \beta[44,45]$. Panax notoginseng can regulate the expression of $\mathrm{AD}$-related circRNAs to achieve the therapeutic effect on AD [46]. Gypenosides can significantly improve learning ability and memory in rats with LPS-induced brain dysfunction [47]. Icariin prevents amyloid beta-induced apoptosis via the PI3K/Akt pathway in PC-12 cells [48] and improves synaptic plasticity through the BDNF/TrkB/Akt pathway [49]. Alpinia Oxyphylla can improve spatial memory performance and downregulated expressions of $\beta$-secretase and accumulation of $A \beta_{1-42}$ in brain tissues [50]. The root bark of Morus alba, as well as its isolated compounds, has shown the potent AChE-, BChE-, and BACE1-inhibitory activities [51]. However, the active ingredients of YZG remain unclear.

Although traditional Chinese medicine contains complex chemical components, only the compounds that can be absorbed into the blood can produce effects. Traditional Chinese medicine is mostly administered orally, and its active substances must be transported to the action target through the blood so as to have an effect. Therefore, the components contained in the serum after administration are the direct-acting substances of traditional Chinese medicine in vivo. Cynomolgus monkeys are nonhuman primates and are similar to humans in genetics and pathophysiology; thus, they are a useful preclinical model $[17,18]$. Therefore, we used this model to evaluate YZG. And the serum pharmacochemistry method was used to analyze and identify the components contained in the serum after oral administration of YZG in the cynomolgus monkeys.

In this study, a total of 63 compounds were determined from YZG using the UPLC/ESI-Q-TOF MS method; most of them were saponins come from Panax ginseng and Panax 
notoginseng, indicating that the main components of YZG that played a medicinal role were saponins. Then, we analyzed and identified the prototype components in the serum of cynomolgus monkey after the oral administration of YZG and found 35 prototype components. And we analyzed the cleavage rules of some representative components. This indicated that these compounds are the possible active components of YZG. In order to study whether these compounds really work, we used in vitro experiments to verify and purchased 14 commercially available compounds to do cell experiments; the results show that nine of them have significant pharmacological activities, which proves to a certain extent that the components absorbed into the blood are indeed the active components of YZG, which provides a basis for our later experiments. However, because other absorbed components are challenging to obtain, we did not verify all the absorbed components in this study, which is the deficiency of this experiment, and the follow-up study will find a way to solve this problem.

\section{Conclusions}

A rapid, sensitive, and convenient UPLC/ESI-Q-TOF MS method was established for the simultaneous qualitative analysis of the chemical compositions of YZG and the absorbed components in the plasma cynomolgus monkey. Seventy compounds were detected in YZG, and 63 compounds of these were identified including 60 saponins, 2 flavones, and 1 methyl ester. In vivo studies showed 33 saponins, 1 flavone, and 1 methyl ester in the plasma of cynomolgus monkeys. The PC12 cell damage model used A $\beta 25-35$ to evaluate the neuroprotective effects of the absorbed components. The results showed that 9 compounds have protective effects: ginsenoside $\mathrm{Rb} 1$, ginsenoside $\mathrm{Rb} 2$, ginsenoside $\mathrm{Rd}$, ginsenoside $\mathrm{Re}$, notoginsenoside $\mathrm{Fa}$, notoginsenoside $\mathrm{R} 1$, notoginsenoside R2, gypenoside A, and gypenoside XLIX. Most of these active components are saponins.

\section{Abbreviations}

YZG:
TCM:
UPLC/ESI-Q-
TOF MS:

$\mathrm{AD}:$

PCA:

OPLS-DA:

DMSO:

SP:

$\mathrm{A} \beta$ :

NFTs:
Yizhi Granule

Traditional Chinese medicine

Ultraperformance liquid

chromatography/electrospray ionization quadrupole time-of-flight mass spectrometry

Alzheimer's disease

Principal component analysis

Orthogonal partial least square

discrimination analysis

Dimethyl sulfoxide

Senile plaque

$\beta$-Amyloid

Neurofibrillary tangles.

\section{Data Availability}

The data used to support the findings of this study are included within the article.

\section{Conflicts of Interest}

The authors declare no conflicts of interest.

\section{Authors' Contributions}

Erwei Hao, Jianfeng Qin, and Wei Wei contributed equally to this work.

\section{Acknowledgments}

This research was funded by the Scientific Research and Technology Development Program of Guangxi (Grant nos. GKG1355004-11, 15-140-31, 17-259-20, 2017AD19024, and AD17195025). The authors thank LetPub (http://www. letpub.com) for its linguistic assistance during the preparation of this manuscript.

\section{References}

[1] G. J. Demakis, "Disability in alzheimer's disease: causes, consequences, and economic considerations," Journal of Health and Human Services Administration, vol. 30, no. 3, pp. 292-305, 2007.

[2] P. Gillespie, E. O'Shea, J. Cullinan et al., "The effects of dependence and function on costs of care for alzheimer's disease and mild cognitive impairment in Ireland," International Journal of Geriatric Psychiatry, vol. 28, no. 3, pp. 256-264, 2013.

[3] S. Freude, M. M. Hettich, C. Schumann et al., "Neuronal IGF1 resistance reduces $\mathrm{A} \beta$ accumulation and protects against premature death in a model of alzheimer's disease," The FASEB Journal, vol. 23, no. 10, pp. 3315-3324, 2009.

[4] J. Jia, F. Wang, C. Wei et al., "The prevalence of dementia in urban and rural areas of China," Alzheimer's \& Dementia, vol. 10, no. 1, pp. 1-9, 2014.

[5] J. Jia, C. Wei, S. Chen et al., “The cost of alzheimer's disease in China and re-estimation of costs worldwide," Alzheimer's \& Dementia, vol. 14, no. 4, pp. 483-491, 2018.

[6] D. J. Selkoe and J. Hardy, "The amyloid hypothesis of alzheimer's disease at 25 years," EMBO Molecular Medicine, vol. 8, no. 6, pp. 595-608, 2016.

[7] J. Li, Y. Liu, W. Li et al., "Metabolic profiling of the effects of ginsenoside re in an alzheimer's disease mouse model," Behavioural Brain Research, vol. 337, p. 160, 2018.

[8] R. Aalinkeel, H. L. Kutscher, A. Singh et al., "Neuroprotective effects of a biodegradable poly(lactic-co-glycolic acid)-ginsenoside rg3 nanoformulation: a potential nanotherapy for alzheimer's disease?" Journal of Drug Targeting, vol. 26, no. 2, pp. 1-28, 2017.

[9] J. Zhao, S. Lu, H. Yu, S. Duan, and J. Zhao, "Baicalin and ginsenoside $r b 1$ promote the proliferation and differentiation of neural stem cells in alzheimer's disease model rats," Brain Research, vol. 1678, pp. 187-194, 2018.

[10] R. Shi, S. Zhang, G. Cheng, X. Yang, N. Zhao, and C. Chen, "Ginsenoside rg1 and acori graminei rhizoma attenuates neuron cell apoptosis by promoting the expression of mir873-5p in alzheimer's disease," Neurochemical Research, vol. 43, no. 8, pp. 1529-1538, 2018.

[11] D. Ye Hong, M. Fu, Y. T. Wang et al., "Neuroprotective effects of ginsenoside $\mathrm{rf}$ on amyloid- $\beta$-induced neurotoxicity in vitro and in vivo," Journal of Alzheimer's Disease, vol. 64, no. 2, pp. 1-14, 2018. 
[12] Z. C. Du and J. G. Deng, "Effect of compound jiaogulan yizhi granules for improving memory in mice," Chinese Journal of Experimental Traditional Medical Formulae, vol. 18, no. 15, pp. 250-253, 2012.

[13] Z. C. Du, E. W. Hao, Q. Huang et al., "Study on the acute toxicological and ubchronic toxicological test of compound jiaogulan yizhi granules," Contemporary Medical Physiology, vol. 18 , no. 19, pp. 1-3, 2012.

[14] Z. C. Du and J. G. Deng, "Clinical observation of compound jaogulan granules on memory improvement in human," Chinese Journal of Experimental Traditional Medical Formulae, vol. 18, no. 12, pp. 262-265, 2012.

[15] E. W. Hao, J. G. Deng, Z. C. Du et al., "Neuroprotective effect research of compound yizhi granule on the pc12 cell injury model induced by a $\beta$-(25-35)," World Chinese Medicine, vol. 11, no. 11, pp. 2227-2230, 2016.

[16] X. W. Yang, "Discovery strategy for effective and active constituents of Chinese material medica based on processes of metabolism and disposition in intra-body," China Journal of Chinese Materia Medica, vol. 32, no. 5, p. 365, 2007.

[17] Z. T. Yang, A. M. Liu, F. Liu et al., "Species-related stability difference of simvastatin in human, cynomolgus monkey and rat plasma," Chinese Journal of Modern Applied Pharmacy, vol. 26, no. 4, pp. 261-264, 2009.

[18] E. Buse, "Development of the immune system in the cynomolgus monkey: the appropriate model in human targeted toxicology?" Journal of Immunotoxicology, vol. 2, no. 4, pp. 211-216, 2005.

[19] Y.-N. Tang, Y.-X. Pang, X.-C. He et al., "UPLC-QTOF-MS identification of metabolites in rat biosamples after oral administration of dioscorea saponins: a comparative study," Journal of Ethnopharmacology, vol. 165, pp. 127-140, 2015.

[20] J.-F. Feng, Y.-N. Tang, H. Ji, Z.-G. Xiao, L. Zhu, and T. Yi, "Biotransformation of Dioscorea nipponica by rat intestinal microflora and cardioprotective effects of diosgenin," Oxidative Medicine and Cellular Longevity, vol. 2017, Article ID 4176518, 9 pages, 2017.

[21] T. Yi, L. Zhu, Y.-N. Tang et al., “An integrated strategy based on UPLC-DAD-QTOF-MS for metabolism and pharmacokinetic studies of herbal medicines: Tibetan "snow lotus" herb (Saussurea laniceps), a case study," Journal of Ethnopharmacology, vol. 153, no. 3, pp. 701-713, 2014.

[22] T. Yi, H. B. Chen, Z.-Z. Zhao, Z.-H. Jiang, S.-Q. Cai, and T.-M. Wang, "Comparative analysis of the major constituents in the traditional Tibetan medicinal plants Saussurea laniceps and S. medusa by LC-DAD-MS," Chromatographia, vol. 70, no. 5-6, pp. 957-962, 2009.

[23] P. Liu, H.-S. Yu, L.-J. Zhang et al., "A rapid method for chemical fingerprint analysis of Pan Panax notoginseng powders by ultra performance liquid chromatography coupled with quadrupole time-of-flight mass spectrometry," Chinese Journal of Natural Medicines, vol. 13, no. 6, pp. 471-480, 2015.

[24] E. M. Sánchez-Salcedo, M. Tassotti, D. Del Rio, F. Hernández, J. J. Martínez, and P. Mena, "(Poly)phenolic fingerprint and chemometric analysis of white (Morus alba L.) and black (Morus nigra L.) mulberry leaves by using a non-targeted UHPLC-MS approach," Food Chemistry, vol. 212, pp. 250255, 2016.

[25] S.-S. Li, Q. Wu, D.-D. Yin, C.-Y. Feng, Z.-A. Liu, and L.-S. Wang, "Phytochemical variation among the traditional Chinese medicine mu dan pi from paeonia suffruticosa (tree peony)," Phytochemistry, vol. 146, pp. 16-24, 2018.
[26] H. Y. Liu, Y. Q. Xu, T. Ouyang et al., "Identification of saponins in yiqi fumai freeze-dried powder for injection by HPLC-Q-TOF-MS," Chinese Journal of Experimental Traditional Medical Formulae, vol. 24, no. 5, pp. 7-12, 2018.

[27] L. Yang, Y. Miao, H. J. Yin et al., "Identification of ginsenosides from Panax quinquefolius in xinyue capsula by LCESI-MS"," Chinese Traditional and Herbal Drugs, vol. 41, no. 12, pp. 1942-1947, 2010.

[28] F. L. Xu, Q. Y. Zhang, L. Jiang et al., "Study on chemical constituents of Radix ginseng destillata alcohol extract by UHPLC-Q-TOF/MS," Traditional Chinese Drug Research and Clinical Pharmacology, vol. 4, no. 26, pp. 529-534, 2015.

[29] W. Xu, J.-h. Zhang, X. Liu et al., "Two new dammarane-type triterpenoid saponins from ginseng medicinal fungal substance," Natural Product Research, vol. 31, no. 10, pp. 1107-1112, 2017.

[30] J.-Y. Wan, C.-Z. Wang, Z. Liu et al., "Determination of American ginseng saponins and their metabolites in human plasma, urine and feces samples by liquid chromatography coupled with quadrupole time-of-flight mass spectrometry," Journal of Chromatography B, vol. 1015-1016, pp. 62-73, 2016.

[31] Z. Jing, Z. X. Qin, P. Bing et al., "Fragmentation pathway of ginsenosides in panaxnotoginseng using electrospray ionization-quadrupole/time-of-flight mass spectrometer," Journal of Chinese Mass Spectrometry Society, vol. 38, no. 1, pp. 97$108,2017$.

[32] K. Zou, S. Zhu, C. Tohda, fnm Cai, and K. Komatsu, "Dammarane-type triterpene saponins from panaxjaponicus," Journal of Natural Products, vol. 65, no. 3, pp. 346-351, 2002.

[33] Y. Chen, Z. Zhao, H. Chen, T. Yi, M. Qin, and Z. Liang, "Chemical differentiation and quality evaluation of commercial asian and american ginsengs based on a UHPLCQTOF/MS/MS metabolomics approach," Phytochemical Analysis, vol. 26, no. 2, pp. 145-160, 2015.

[34] S. Hui, L. Chang, A. H. Zhang et al., "Rapid discovery and global characterization of multiple constituents from kai-xinsan using an integrated mse data acquisition mode strategy based on ultra-performance liquid chromatography coupled to electrospray ionization/quadrupole-time-of-flight mass," Analytical Methods, vol. 7, no. 1, pp. 279-286, 2014.

[35] J.-Q. Liu, C.-F. Wang, J.-C. Chen et al., "Six new triterpenoid glycosides from Gynostemma pentaphyllum," Helvetica Chimica Acta, vol. 92, no. 12, pp. 2737-2745, 2009.

[36] Y. Feng and L. H. Hu, "Studies on chemical constituents of jiaogulan (Gynostemma pentaphyllum)," ACS Symposium Series, vol. 925, pp. 170-184, 2006.

[37] Z. M. Lv, "Advances in research on genes related to betaamyloid metabolism and alzheimer's disease," Journal of International Neurology and Neurosurgery, vol. 34, no. 6, pp. 527-530, 2007.

[38] K. Youn, S. Lee, W.-S. Jeong, C.-T. Ho, and M. Jun, "Protective role of corilagin on A $\beta 25-35$-induced neurotoxicity: suppression of NF- $\kappa \mathrm{B}$ signaling pathway," Journal of Medicinal Food, vol. 19, no. 10, pp. 901-911, 2016.

[39] R. Sultana, P. Mecocci, F. Mangialasche, R. Cecchetti, M. Baglioni, and D. A. Butterfield, "Increased protein and lipid oxidative damage in mitochondria isolated from lymphocytes from patients with alzheimer's disease: insights into the role of oxidative stress in alzheimer's disease and initial investigations into a potential biomarker for this dementing disorder," Journal of Alzheimer's Disease, vol. 24, no. 1, pp. 77-84, 2011. 
[40] M. Hüll, M. Berger, J. Bauer et al., "Inflammatory mechanisms in alzheimer's disease," European Archives of Psychiatry and Clinical Neuroscience, vol. 246, no. 3, pp. 124-128, 1994.

[41] P. Mcgeer and E. Mcgeer, "The inflammatory response system of brain: implications for therapy of alzheimer and other neurodegenerative diseases," Brain Research Reviews, vol. 21, no. 2, pp. 195-218, 1995.

[42] G. Esposito, D. De Filippis, M. C. Maiuri, D. De Stefano, R. Carnuccio, and T. Iuvone, "Cannabidiol inhibits inducible nitric oxide synthase protein expression and nitric oxide production in $\beta$-amyloid stimulated PC12 neurons through p38 MAP kinase and NF- $\kappa \mathrm{B}$ involvement," Neuroscience Letters, vol. 399, no. 1-2, pp. 91-95, 2006.

[43] A. Nordberg, "Mechanisms behind the neuroprotective actions of cholinesterase inhibitors in alzheimer disease," Alzheimer Disease \& Associated Disorders, vol. 20, no. 1, p. S12, 2006.

[44] H.-J. Kim, S.-W. Jung, S.-Y. Kim et al., "Panax ginseng as an adjuvant treatment for alzheimer's disease," Journal of Ginseng Research, vol. 42, no. 4, pp. 401-411, 2018.

[45] M. Razgonova, V. Veselov, A. Zakharenko et al., "Panax ginseng components and the pathogenesis of alzheimer's disease (review)," Molecular Medicine Reports, vol. 19, pp. 2975-2998, 2019.

[46] J.-L. Huang, Z.-H. Xu, S.-M. Yang et al., "Identification of differentially expressed profiles of alzheimer's disease associated circular RNAs in a panax notoginseng saponins-treated alzheimer's disease mouse model," Computational and Structural Biotechnology Journal, vol. 16, pp. 523-531, 2018.

[47] B. Lee, I. Shim, H. Lee, and D.-H. Hahm, "Gypenosides attenuate lipopolysaccharide-induced neuroinflammation and anxiety-like behaviors in rats," Animal Cells and Systems, vol. 22, no. 5, pp. 305-316, 2018.

[48] D. Zhang, Z. Wang, C. Sheng et al., "Icariin prevents amyloid beta-induced apoptosis via the pi3k/akt pathway in pc-12 cells," Evidence-Based Complementary and Alternative Medicine, vol. 2015, Article ID 235265, 9 pages, 2015.

[49] C. X. Sheng, P. P. Xu, K. X. Zhou, D. Deng, C. Zhang, and $\mathrm{Z}$. Wang, "Icariin attenuates synaptic and cognitive deficits in an A $\beta 1-42$-induced rat model of alzheimer's disease," BioMed Research International, vol. 2017, Article ID 7464872, 12 pages, 2017.

[50] B. S. He, F. X. Xu, T. X. Yan et al., "Tectochrysin from Alpinia oxyphylla miq. Alleviates A $\beta 1-42$ induced learning and memory impairments in mice," European Journal of Pharmacology, vol. 842, pp. 365-372, 2018.

[51] E. B. Kuk, A. R. Jo, S. I. Oh et al., "Anti-alzheimer's disease activity of compounds from the root bark of Morus alba L," Archives of Pharmacal Research, vol. 40, no. 3, pp. 338-349, 2017. 